ESAIM: COCV 20 (2014) 488-516

DOI: $10.1051 / \mathrm{cocv} / 2013072$
ESAIM: Control, Optimisation and Calculus of Variations

www.esaim-cocv.org

\title{
ADAPTIVE STABILIZATION OF COUPLED PDE-ODE SYSTEMS WITH MULTIPLE UNCERTAINTIES *
}

\author{
JiAN LI $^{1,2}$ AND YUNGANG LIU ${ }^{1}$
}

\begin{abstract}
The adaptive stabilization is investigated for a class of coupled PDE-ODE systems with multiple uncertainties. The presence of the multiple uncertainties and the interaction between the subsystems makes the systems to be considered more general and representative, and moreover it may result in the ineffectiveness of the conventional methods on this topic. Motivated by the existing literature, an infinite-dimensional backsteppping transformation with new kernel functions is first introduced to change the original system into a target system, from which the control design and performance analysis of the original system will become quite convenient. Then, by certainty equivalence principle and Lyapunov method, an adaptive stabilizing controller is successfully constructed, which guarantees that all the closed-loop system states are bounded while the original system states converging to zero. A simulation example is provided to validate the proposed method.
\end{abstract}

Mathematics Subject Classification. 93C20, 93D15, 93D21.

Received August 27, 2012. Revised August 7, 2013.

Published online March 14, 2014.

\section{INTRODUCTION}

The present paper is about the controls of ordinary differential equations (ODEs) with infinite-dimensional actuator dynamics governed by partial differential equations (PDEs). Such problems have attracted a lot of attention recently (see e.g., [1-6] and the references therein), and particularly, the stabilization of ODEs with diffusion-dominated actuator dynamics has been investigated in [4-6]. It is worth pointing out that the systems investigated in [4-6] are limited by the basic hypothesis that there only exists the action from dynamic actuator (i.e., PDE sub-system) to plant (i.e., ODE sub-system). However, in some cases, the opposing action from plant to dynamic actuator is a bit intense and cannot be ignored, which makes the systems strongly coupled and more complex, and consequently, if no additional treatments are carried out, the system performance would become unacceptable.

\footnotetext{
Keywords and phrases. Coupled PDE-ODE systems, spatially varying coefficient, adaptive stabilization, infinite-dimensional backstepping.

* This work was supported by the National Natural Science Foundation of China $(61233014,61273084)$, the Natural Science Foundation for Distinguished Young Scholar of Shandong Province of China (JQ200919) and the Independent Innovation Foundation of Shandong University (2012JC014).

1 School of Control Science and Engineering, Shandong University, Jinan 250061, P.R. China. lygfr@sdu.edu.cn

2 School of Mathematics and Information Science, Yantai University, Yantai 264005, P.R. China.
} 
As a matter of fact, the controls of coupled PDE-ODE systems have received continuous investigation over the last decades (see e.g., [7-17] and the references therein). Recently, the stabilization of ODE systems coupled by a parabolic PDE has been investigated in $[7,8]$, but the systems considered are required to be precisely known, i.e., no uncertainties/unknowns exist in the systems. However, the accurate model of the controlled systems is usually difficult to obtain in practice, even impossible. Moreover, the presence of the uncertainties/unknowns will make the methods and ideas used in the literature inapplicable to stabilize the uncertain systems. Consequently, how to stabilize the coupled PDE-ODE systems with uncertainties becomes a much practical and challenge problem, and to the best of our knowledge, no research has been found on this topic.

This paper is devoted to the stabilization of a class of strongly coupled PDE-ODE systems with multiple uncertainties, where the PDE sub-system is a diffusion equation with unknown diffusion coefficient and the ODE sub-system is a linear time-invariant system with system matrix and input matrix being parameterized by an unknown vector. A distinguished feature of the systems under discussion is that in PDE sub-system, there exists intense action from the ODE sub-system, and the action possesses an unknown spatially varying coefficient (called influence function). Mainly due to the presence of the strong coupling and multiple uncertainties, the systems in present paper are more general than those in the related literature [5-7]. Specifically, in $[5,6]$, there is only the action from the PDE sub-system to the ODE one and no opposing action, and moreover, only the diffusion coefficient permits perturbations or uncertainties. In [7], although both the action from the PDE sub-system to the ODE one and the opposing action are considered, all the system parameters (e.g., diffusion coefficient) are precisely known. In order to stabilize the systems under discussion, it is necessary to effectively reject the unexpected effects caused by the coupling and uncertainties. For this, infinite-dimensional backstepping method, adaptive technique and certainty equivalence principle are adopted to construct an adaptive state-feedback controller which guarantees that the original system states converge to zero while the other closed-loop system states keeping bounded.

For clarity, the main contributions of the paper are highlighted as follows:

(i) The action from the ODE sub-system to the PDE one is intense and cannot be ignored, which does not exist in the closely related works [5,6]. The presence of such action makes the system under discussion strongly coupled and more complicated than those of $[5,6]$, and hence makes the kernel functions of the infinite-dimensional backstepping transformation more difficult to derive.

(ii) Unlike [5-7], multiple uncertainties are involved in the systems under discussion. This renders the system under discussion more general and representative. On the other hand, to cope with these uncertainties, additional dynamic compensations should be made, which will make the design of desired controller more difficult, specially, the derivation of the desirable target system.

The remainder of the paper is organized as follows. Section 2 formulates the system model and the control objective. Section 3 presents the adaptive control design. Section 4 provides the rigorous proof for the stability of the closed-loop system. Section 5 gives a numerical example to illustrate the effectiveness of the proposed method. Section 6 addresses some concluding remarks. The paper ends with an appendix that collects the proofs of some important propositions and several useful inequalities.

Notations. Throughout the paper, $\|\cdot\|$ denotes the Euclidean norm for column vectors or the corresponding induced norm for matrices. $I$ denotes the identity matrix with appropriate dimension. $\lambda_{\min }(P)$ denotes the minimal eigenvalue of square matrix $P$. Let $S_{K}=\sup _{\theta \in \Omega}\|K(\theta)\|$ for continuous matrix function $K(\theta)$ defined on the set $\Omega$ concerned in present paper. The projector operator in $\dot{\hat{\zeta}}(t)=\operatorname{Proj}_{[\underline{\zeta}, \bar{\zeta}]}\{\tau\}$ is defined as

$$
\operatorname{Proj}_{[\underline{\zeta}, \bar{\zeta}]}\{\tau\}= \begin{cases}0, & \hat{\zeta}=\zeta \text { and } \tau<0 \\ 0, & \hat{\zeta}=\overline{\bar{\zeta}} \text { and } \tau>0 \\ \tau, & \text { else. }\end{cases}
$$




\section{Problem Formulation}

In this paper, we consider the following coupled system consisting of an ODE and a PDE:

$$
\left\{\begin{array}{l}
\dot{X}(t)=A(\theta) X(t)+B(\theta) u(0, t) \\
u_{t}(x, t)=\varepsilon u_{x x}(x, t)+\lambda(x) C X(t), \\
u_{x}(0, t)=0 \\
u(D, t)=U(t)
\end{array}\right.
$$

where $X(t) \in \mathbb{R}^{n}$ with initial value $X(0)=X_{0}$ is the vector state of the ODE sub-system and $u(x, t)$ with initial value $u(x, 0)=u_{0}(x)$ is the scalar state of the PDE sub-system; $U(t)$ is the scale input to the entire system; $u_{t}=\frac{\partial u}{\partial t}, u_{x}=\frac{\partial u}{\partial x}$ and $u_{x x}=\frac{\partial^{2} u}{\partial x^{2}} ; \varepsilon$ is an unknown positive constant, called diffusion coefficient; $\lambda(x)$ is an unknown continuous function defined on $[0, D]$, called influence function; $D$ is an arbitrary positive constant which denotes the length of the PDE domain; $C \in \mathbb{R}^{1 \times n}$ is a known constant matrix, $A(\theta)$ and $B(\theta)$ are linearly parameterized with unknown constant vector $\theta=\left[\theta_{1}, \ldots, \theta_{p}\right]^{\mathrm{T}}$, i.e.,

$$
A(\theta)=A_{0}+\sum_{i=1}^{p} \theta_{i} A_{i}, \quad B(\theta)=B_{0}+\sum_{i=1}^{p} \theta_{i} B_{i},
$$

where $p$ is a positive integer, $\theta_{i}$ 's are unknown constants, $A_{i} \in \mathbb{R}^{n \times n}, i=0, \ldots, p$ and $B_{i} \in \mathbb{R}^{1 \times n}, i=0, \ldots, p$ are known constant matrices.

The main objective of the paper is to design an adaptive state-feedback controller such that all the states of closed-loop system are bounded while the states of system (2.1) converging to zero. To make this feasible, the following assumptions are made on system (2.1):

Assumption 2.1. There exist known constants $\underline{\theta}_{i}, \bar{\theta}_{i}(i=1, \ldots, p), \underline{\varepsilon}, \underline{\lambda}$ and $\bar{\lambda}$ such that

$$
\left\{\begin{array}{l}
\underline{\theta}_{i} \leq \theta_{i} \leq \bar{\theta}_{i}, i=1, \ldots, p \\
\varepsilon \geq \underline{\varepsilon}>0 \\
\underline{\lambda} \leq \lambda(x) \leq \bar{\lambda}, \forall x \in[0, D] .
\end{array}\right.
$$

Assumption 2.2. For any $\theta \in \Omega=\left[\underline{\theta}_{1}, \bar{\theta}_{1}\right] \times \cdots \times\left[\underline{\theta}_{p}, \bar{\theta}_{p}\right]$, the pair $(A(\theta), B(\theta))$ is controllable, and there exist continuous matrix function $Q(\theta)=Q(\theta)^{\mathrm{T}}>0$ and continuously differentiable matrix functions $K(\theta)$, $P(\theta)=P(\theta)^{\mathrm{T}}>0$ such that

$$
(A(\theta)+B(\theta) K(\theta))^{\mathrm{T}} P(\theta)+P(\theta)(A(\theta)+B(\theta) K(\theta))=-Q(\theta) .
$$

Moreover, $S_{P}\left(\right.$ i.e., $\left.\sup _{\theta \in \Omega}\|P(\theta)\|\right), \inf _{\theta \in \Omega} \lambda_{\min }(Q(\theta))$ and $\inf _{\theta \in \Omega} \lambda_{\min }(P(\theta))$ exist and are known.

We make a remark on system (2.1) to end this section.

Remark 2.3. Although the ODE subsystem in (2.1) is the same as that in [2], the actuator dynamics in (2.1) are described by a diffusion equation, rather than a first-order hyperbolic PDEs in [2]. Moreover, in the present paper, the actuator and the controlled system are mutually coupled, whereas in [2], there is only action from the actuator to the controlled system. These make the control design in our paper more difficult than that of [2].

\section{Adaptive Control Design}

This section is to design adaptive state-feedback controller for system (2.1). First, an infinite-dimensional backstepping transformation is introduced to transform the original system (2.1) into a new one (called target 
system). Then, it is proven that the stability of the target system implies the desirable stability of the original system under the same controller. Finally, an adaptive controller is successfully constructed by certainty equivalence principle and related adaptive techniques.

Let $\hat{\theta}_{i}(t), i=1, \ldots, p, \hat{\varepsilon}(t)$ and $\hat{\lambda}(x, t)$ denote the dynamic compensations to the unknown parameters $\theta_{i}, i=$ $1, \ldots, p, \varepsilon$ and $\lambda(x)$, respectively, and satisfy for any $t \geq 0$,

$$
\left\{\begin{array}{l}
\underline{\theta}_{i} \leq \hat{\theta}_{i}(t) \leq \bar{\theta}_{i}, i=1, \ldots, p \\
\hat{\varepsilon}(t) \geq \underline{\varepsilon}>0 \\
\underline{\lambda} \leq \hat{\lambda}(x, t) \leq \bar{\lambda}, \forall x \in[0, D]
\end{array}\right.
$$

The dynamic updating laws that $\hat{\theta}_{i}(t), i=1, \ldots, p, \hat{\varepsilon}(t)$ and $\hat{\lambda}(x, t)$ obey will be determined later. We then define

$$
\left\{\begin{aligned}
\gamma_{0}(x, t)= & K(\hat{\theta})-\frac{1}{\hat{\varepsilon}(t)} \int_{0}^{x} \int_{0}^{\eta} \hat{\lambda}(\xi, t) C \mathrm{~d} \xi \mathrm{d} \eta \\
\gamma_{i+1}(x, t)= & \frac{1}{\hat{\varepsilon}(t)} \int_{0}^{x} \int_{0}^{\eta} \gamma_{i}(\xi, t) A(\hat{\theta}) \mathrm{d} \xi \mathrm{d} \eta \\
& +\frac{1}{2 \hat{\varepsilon}(t)^{2}} \int_{0}^{x} \int_{0}^{x-y}(x-y-\xi)^{2} \gamma_{i}(\xi, t) B(\hat{\theta}) \mathrm{d} \xi \hat{\lambda}(y, t) C \mathrm{~d} y, i=0,1,2, \ldots
\end{aligned}\right.
$$

where $\hat{\theta}(t)=\left[\hat{\theta}_{1}(t), \cdots, \hat{\theta}_{p}(t)\right]^{\mathrm{T}}, K(\hat{\theta}) \in \mathbb{R}^{1 \times n}$ is chosen such that $A(\hat{\theta})+B(\hat{\theta}) K(\hat{\theta})$ is Hurwitz for each $\hat{\theta} \in \Omega$.

Thus, for system (2.1), we introduce the following infinite-dimensional backstepping transformation:

$$
w(x, t)=u(x, t)-\int_{0}^{x} k(x, y, t) u(y, t) \mathrm{d} y-\gamma(x, t) X(t),
$$

where

$$
\left\{\begin{array}{l}
\gamma(x, t)=\sum_{i=0}^{+\infty} \gamma_{i}(x, t) \\
k(x, y, t)=\frac{1}{\hat{\varepsilon}(t)} \int_{0}^{x-y} \gamma(\xi, t) B(\hat{\theta}) \mathrm{d} \xi
\end{array}\right.
$$

From (3.3), one can see that kernel functions $k(\cdot)$ and $\gamma(\cdot)$ of backstepping transformation (3.2) are different from those of [5-7]. Specifically, due to the presence of the dynamic compensations to the unknown parameters, the kernel functions in (3.2) are time-varying and hence essentially different from the time-invariant case of $[5,7]$. Moreover, multiple uncertainties and strong coupling in system (2.1) make the kernel functions of (3.2) more complicated than those of [6] where single uncertainty is considered and no coupling exists between sub-systems.

From (3.1) and (3.3), it can be seen that $\gamma(\cdot)$ and $k(\cdot)$ respectively satisfy

$$
\left\{\begin{array}{l}
\hat{\varepsilon}(t) \gamma_{x x}(x, t)=-\hat{\lambda}(x, t) C+\gamma(x, t) A(\hat{\theta})+\frac{1}{\hat{\varepsilon}(t)} \int_{0}^{x} \int_{0}^{x-y} \gamma(\xi, t) B(\hat{\theta}) \mathrm{d} \xi \hat{\lambda}(y, t) C \mathrm{~d} y \\
\gamma_{x}(0, t)=0 \\
\gamma(0, t)=K(\hat{\theta})
\end{array}\right.
$$

and

$$
\left\{\begin{array}{l}
k_{x x}(x, y, t)=k_{y y}(x, y, t) \\
k(x, x, t)=0 \\
k_{y}(x, 0, t)=-\frac{1}{\hat{\varepsilon}(t)} \gamma(x, t) B(\hat{\theta})
\end{array}\right.
$$

which will be used later in deriving of the desirable target system.

It is necessary to point out that, an inverse transformation exists for (3.2), which is described as follows:

$$
u(x, t)=w(x, t)+\int_{0}^{x} l(x, y, t) w(y, t) \mathrm{d} y+\beta(x, t) X(t),
$$


where

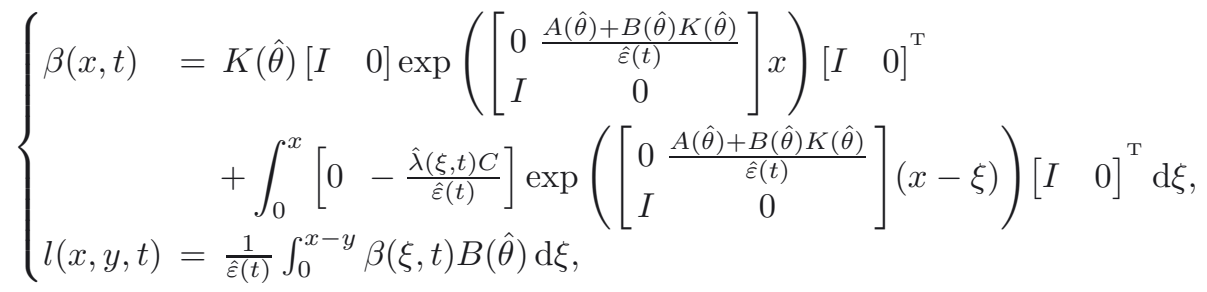

satisfy the following equations:

$$
\left\{\begin{array}{l}
\hat{\varepsilon}(t) \beta_{x x}(x, t)=-\hat{\lambda}(x, t) C+\beta(x, t)(A(\hat{\theta})+B(\hat{\theta}) K(\hat{\theta})), \\
\beta_{x}(0, t)=0, \\
\beta(0, t)=K(\hat{\theta}),
\end{array}\right.
$$

and

$$
\left\{\begin{array}{l}
l_{x x}(x, y, t)=l_{y y}(x, y, t), \\
l(x, x, t)=0, \\
l_{y}(x, 0, t)=-\frac{1}{\hat{\varepsilon}(t)} \beta(x, t) B(\hat{\theta}) .
\end{array}\right.
$$

For the kernel functions of the backstepping transformation (3.2) and its inverse transformation (3.6) defined in (3.3) and (3.7), respectively, the following properties will be used in the subsequent adaptive control design and stability analysis of the closed-loop system.

Proposition 3.1. The kernel functions $k(x, y, t), \gamma(x, t)$ and $l(x, y, t), \beta(x, t)$ defined in (3.3) and (3.7), respectively, are bounded on their separate domains of definition, and so are their partial derivatives with respect to $x$. Moreover, there hold the following equations:

$$
\left\{\begin{array}{l}
\int_{y}^{x} k(x, \xi, t) l(\xi, y, t) \mathrm{d} \xi=l(x, y, t)-k(x, y, t), \\
\int_{0}^{x} k(x, y, t) \beta(y, t) \mathrm{d} y=\beta(x, t)-\gamma(x, t) .
\end{array}\right.
$$

Proof. See part A of Appendix in the paper.

From the above proposition, we know that

$$
\left\{\begin{array}{l}
\sup _{0 \leq x \leq D, t \geq 0}\|\gamma(x, t)\| \leq M_{1}, \sup _{0 \leq y \leq x \leq D, t \geq 0}|k(x, y, t)| \leq M_{1}, \\
\sup _{0 \leq x \leq D, t \geq 0}\|\beta(x, t)\| \leq M_{1}, \sup _{0 \leq y \leq x \leq D, t \geq 0}|l(x, y, t)| \leq M_{1}, \\
\sup _{0 \leq x \leq D, t \geq 0}\left\|\gamma_{x}(x, t)\right\| \leq M_{1}, \sup _{0 \leq y \leq x \leq D, t \geq 0}\left|k_{x}(x, y, t)\right| \leq M_{1}, \\
\sup _{0 \leq x \leq D, t \geq 0}\left\|\beta_{x}(x, t)\right\| \leq M_{1}, \sup _{0 \leq y \leq x \leq D, t \geq 0}\left|l_{x}(x, y, t)\right| \leq M_{1},
\end{array}\right.
$$

where $M_{1}$ is a positive constant whose one suitable value is given in the proof of Proposition 3.1.

By transformation (3.2) and its inverse transformation (3.6), a new system (called target system) can be obtained from original system (2.1), which is shown in the following proposition and will make the control design and performance analysis of the original system become much convenient. 
Proposition 3.2. System (2.1) can be changed into the following target system under transformation (3.2) and its inverse transformation (3.6):

$$
\left\{\begin{aligned}
& \dot{X}(t)=(A(\hat{\theta})+B(\hat{\theta}) K(\hat{\theta})) X(t)+(\tilde{A}(\tilde{\theta})+\tilde{B}(\tilde{\theta}) K(\hat{\theta})) X(t)+B(\theta) w(0, t), \\
& w_{t}(x, t)= \varepsilon w_{x x}(x, t)+\int_{0}^{x} w(y, t) \varphi_{1}(x, y, t) \mathrm{d} y+\varphi_{2}(x, t) X(t) \\
&+\left(\tilde{\lambda}(x, t)-\int_{0}^{x} \tilde{\lambda}(y, t) k(x, y, t) \mathrm{d} y\right) C X(t)+\tilde{\varepsilon}(t) \varphi_{3}(x, t) \\
&-\gamma(x, t)(\tilde{B}(\tilde{\theta}) w(0, t)+(\tilde{A}(\tilde{\theta})+\tilde{B}(\tilde{\theta}) K(\hat{\theta})) X(t)) \\
& w_{x}(0, t)= 0 \\
& w(D, t)= U(t)-\int_{0}^{D} k(D, y, t)\left(w(y, t)+\int_{0}^{y} l(y, \xi, t) w(\xi, t) \mathrm{d} \xi\right) \mathrm{d} y \\
&-\left(\int_{0}^{D} k(D, y, t) \beta(y, t) \mathrm{d} y+\gamma(D, t)\right) X(t) .
\end{aligned}\right.
$$

where $\tilde{\theta}(t)=\theta-\hat{\theta}(t), \tilde{\varepsilon}(t)=\varepsilon-\hat{\varepsilon}(t), \tilde{\lambda}(x, t)=\lambda(x)-\hat{\lambda}(x, t), \tilde{A}(\tilde{\theta})=A(\theta)-A(\hat{\theta}), \tilde{B}(\tilde{\theta})=B(\theta)-B(\hat{\theta})$ and

$$
\left\{\begin{aligned}
\varphi_{1}(x, y, t)= & -l_{t}(x, y, t)+\int_{y}^{x} k(x, \xi, t) l_{t}(\xi, y, t) \mathrm{d} \xi, \\
\varphi_{2}(x, t)= & -\beta_{t}(x, t)+\int_{0}^{x} k(x, y, t) \beta_{t}(y, t) \mathrm{d} y, \\
\varphi_{3}(x, t)= & \frac{1}{\hat{\varepsilon}(t)} \gamma(x, t)((A(\hat{\theta})+B(\hat{\theta}) K(\hat{\theta})) X(t)+B(\hat{\theta}) w(0, t)) \\
& +\frac{1}{\hat{\varepsilon}(t)}\left(\int_{0}^{x} k(x, y, t) \hat{\lambda}(y, t) \mathrm{d} y-\hat{\lambda}\left({ }^{\prime} x, t\right)\right) C X(t) .
\end{aligned}\right.
$$

Proof. See part B of Appendix in the paper.

In fact, it can be proven that once the target system is stabilized in certain sense by a controller, the original system (2.1) would be stabilized in the desirable sense by the same one. This is rigorously claimed in the following lemma.

Lemma 3.3. If there is a controller $U(t)$, which satisfies $\lim _{t \rightarrow+\infty} U(t)=0$ and renders the target system (3.12) stable in the sense:

$$
\left\{\begin{array}{l}
\sup _{t \geq 0} \int_{0}^{D} w_{x}(x, t)^{2} \mathrm{~d} x<+\infty \\
\lim _{t \rightarrow+\infty}\left(\int_{0}^{D} w(x, t)^{2} \mathrm{~d} x+\|X(t)\|^{2}\right)=0
\end{array}\right.
$$

then the original system (2.1) with the same controller $U(t)$ in loop is stable in the sense:

$$
\lim _{t \rightarrow+\infty}(|u(x, t)|+\|X(t)\|)=0 .
$$

Proof. Since $X(t)$ keeps unchanged under the transformation (3.2), we only need to prove $\lim _{t \rightarrow+\infty}|u(x, t)|=0$ for the original system (2.1) with $U(t)$ in the loop. For this, by Agmon's inequality (see Lem. D.3 in part D of Appendix in the paper) and noting $u(D, t)=U(t)$, there would hold

$$
u(x, t)^{2} \leq U(t)^{2}+2 \sqrt{\int_{0}^{D} u(x, t)^{2} \mathrm{~d} x} \sqrt{\int_{0}^{D} u_{x}(x, t)^{2} \mathrm{~d} x} .
$$

Then, it suffices to show that

$$
\lim _{t \rightarrow+\infty} \int_{0}^{D} u(x, t)^{2} \mathrm{~d} x=0, \quad \sup _{t \geq 0} \int_{0}^{D} u_{x}(x, t)^{2} \mathrm{~d} x<+\infty
$$

which, together with the hypothesis $\lim _{t \rightarrow+\infty} U(t)=0$, would imply that $\lim _{t \rightarrow+\infty}|u(x, t)|=0$. 
First, we will prove the former of (3.15). From (3.6), by completing the square and Hölder's inequality ${ }^{3}$, we have

$$
\begin{aligned}
u(x, t)^{2} & \leq 3 w(x, t)^{2}+3\left(\int_{0}^{x} l(x, y, t) w(y, t) \mathrm{d} y\right)^{2}+3\|X(t)\|^{2}\|\beta(x, t)\|^{2} \\
& \leq 3 w(x, t)^{2}+3 \int_{0}^{x} l(x, y, t)^{2} \mathrm{~d} y \int_{0}^{x} w(y, t)^{2} \mathrm{~d} y+3\|X(t)\|^{2}\|\beta(x, t)\|^{2} .
\end{aligned}
$$

Integrating both sides of the above inequality over $[0, D]$ yields

$$
\begin{aligned}
\int_{0}^{D} u(x, t)^{2} \mathrm{~d} x \leq & 3 \int_{0}^{D} w(x, t)^{2} \mathrm{~d} x+3 \int_{0}^{D}\left(\int_{0}^{x} l(x, y, t)^{2} \mathrm{~d} y \int_{0}^{x} w(y, t)^{2} \mathrm{~d} y\right) \mathrm{d} x \\
& +3\|X(t)\|^{2} \int_{0}^{D}\|\beta(x, t)\|^{2} \mathrm{~d} x .
\end{aligned}
$$

By (3.11) and noting that $0 \leq x \leq D$, it follows from the above inequality that

$$
\int_{0}^{D} u(x, t)^{2} \mathrm{~d} x \leq 3\left(1+D^{2} M_{1}^{2}\right) \int_{0}^{D} w(x, t)^{2} \mathrm{~d} x+3 D M_{1}^{2}\|X(t)\|^{2} .
$$

This, together with (3.14), directly concludes $\lim _{t \rightarrow+\infty} \int_{0}^{D} u(x, t)^{2} \mathrm{~d} x=0$.

Second, by computing $u_{x}(x, t)$ from (3.6) and in the similar way to the above, we can obtain

$$
\int_{0}^{D} u_{x}(x, t)^{2} \mathrm{~d} x \leq 3 \int_{0}^{D} w_{x}(x, t)^{2} \mathrm{~d} x+3 D^{2} M_{1}^{2} \int_{0}^{D} w(x, t)^{2} \mathrm{~d} x+3 D M_{1}^{2}\|X(t)\|^{2},
$$

which, together with (3.14), immediately yields the latter of (3.15).

By the above lemma, we conclude that, to stabilize system (2.1) in the desirable sense, it suffices to stabilize target system (3.12) in the sense of (3.14). Thus, we turn to the design of the state-feedback controller for the target system (3.12). In fact, suppose that $\theta, \varepsilon$ and $\lambda(x)$ are known. By letting $\hat{\theta}=\theta, \hat{\varepsilon}=\varepsilon$ and $\hat{\lambda}(x)=\lambda(x)$, and motivated by [7], controller $U(t)$ is chosen as:

$$
U(t)=\int_{0}^{D} k(D, y)\left(w(y, t)+\int_{0}^{y} l(y, \xi) w(\xi, t) \mathrm{d} \xi\right) \mathrm{d} y+\left(\int_{0}^{D} k(D, y) \beta(y) \mathrm{d} y+\gamma(D)\right) X(t) .
$$

In terms of the proof of Theorem 1 in [7], it can be proven that, the target system (3.12) under controller (3.18) is exponentially stable in the sense of norm $\left(\|X(t)\|^{2}+\int_{0}^{D} w(x, t)^{2} \mathrm{~d} x+\int_{0}^{D} w_{x}(x, t)^{2} \mathrm{~d} x\right)^{\frac{1}{2}}$.

However, since $\theta, \varepsilon$ and $\lambda(x)$ are unknown in the paper, the above controller is not applicable. Inspired by the existing works, the following dynamic compensations of the unknown parameters are founded to overcome the unknowns/uncertainties:

$$
\left\{\begin{array}{lll}
\dot{\hat{\varepsilon}}(t) & =\operatorname{Proj}_{[\underline{\varepsilon},+\infty)}\left\{\tau_{\varepsilon}(t)\right\}, \quad \hat{\varepsilon}(0) \geq \underline{\varepsilon}, \\
\hat{\lambda}_{t}(x, t)=\operatorname{Proj}_{[\underline{\lambda}, \bar{\lambda}]}\left\{\tau_{\lambda}(x, t)\right\}, \quad \underline{\lambda} \leq \hat{\lambda}(x, 0) \leq \bar{\lambda}, \forall x \in[0, D], \\
\dot{\hat{\theta}}_{i}(t)=\operatorname{Proj}_{\left[\underline{\theta}_{i}, \bar{\theta}_{i}\right]}\left\{\tau_{\theta_{i}}(t)\right\}, \quad \underline{\theta}_{i} \leq \hat{\theta}_{i}(0) \leq \bar{\theta}_{i}, i=1, \ldots, p,
\end{array}\right.
$$

\footnotetext{
${ }^{3}$ For any functions $f(\cdot)$ and $g(\cdot)$ which are $m$-times and $n$-times integrable on $[a, b]$, respectively, there holds $\int_{a}^{b}|f(x) g(x)| \mathrm{d} x \leq$ $\left(\int_{a}^{b}|f(x)|^{m} \mathrm{~d} x\right)^{\frac{1}{m}} \cdot\left(\int_{a}^{b}|g(x)|^{n} \mathrm{~d} x\right)^{\frac{1}{n}}$, where $1<m, n<+\infty$ satisfy $\frac{1}{m}+\frac{1}{n}=1$.
} 
where

$$
\left\{\begin{array}{l}
\tau_{\varepsilon}(t)=\frac{\sigma \int_{0}^{D} w(x, t) \varphi_{3}(x, t) \mathrm{d} x}{W(t)}, \\
\tau_{\lambda}(x, t)=\frac{\sigma\left(w(x, t)-\int_{x}^{D} w(y, t) k(y, x, t) \mathrm{d} y\right) C X(t)}{W(t)}, \\
\tau_{\theta_{i}}(t)=\frac{2 \sigma X(t)^{\mathrm{T}} P(\hat{\theta})\left(A_{i}+B_{i} K(\hat{\theta})\right) X(t)}{\rho W(t)}-\frac{\sigma \int_{0}^{D} w(x, t) \gamma(x, t) \mathrm{d} x\left(B_{i} w(0, t)+\left(A_{i}+B_{i} K(\hat{\theta})\right) X(t)\right)}{W(t)}, i=1, \ldots, p,
\end{array}\right.
$$

with $\sigma$ and $\rho$ being to-be-determined positive constants,

$$
W(t)=1+2 X(t)^{\mathrm{T}} P(\hat{\theta}) X(t)+\rho \int_{0}^{D} w(x, t)^{2} \mathrm{~d} x
$$

and $P(\hat{\theta})=P(\hat{\theta})^{\mathrm{T}}>0$ being the solution of the following Lyapunov equation

$$
(A(\hat{\theta})+B(\hat{\theta}) K(\hat{\theta}))^{\mathrm{T}} P(\hat{\theta})+P(\hat{\theta})(A(\hat{\theta})+B(\hat{\theta}) K(\hat{\theta}))=-Q(\hat{\theta}),
$$

for some $Q(\hat{\theta})=Q(\hat{\theta})^{\mathrm{T}}>0$.

Then, based on the certainty equivalence principle and the controller designed before (i.e., unknown parameters $\theta, \varepsilon$ and $\lambda(x)$ in (3.18) are replaced by their dynamic estimates $\hat{\theta}, \hat{\varepsilon}$ and $\hat{\lambda}(x)$, respectively), the desirable adaptive state-feedback controller is chosen as:

$$
U(t)=\int_{0}^{D} k(D, y, t)\left(w(y, t)+\int_{0}^{y} l(y, \xi, t) w(\xi, t) \mathrm{d} \xi\right) \mathrm{d} y+\left(\int_{0}^{D} k(D, y, t) \beta(y, t) \mathrm{d} y+\gamma(D, t)\right) X(t)(3.23)
$$

The following two propositions respectively give the properties of the projection operator and the target system, which will be used in the later stability analysis of the closed-loop system.

Proposition 3.4. (see [18]) For the projection operator defined in (1.1), there hold

(i) $\left|\operatorname{Proj}_{[\underline{\zeta}, \bar{\zeta}]}\{\tau\}\right| \leq|\tau|$;

(ii) For $\hat{\zeta}(0) \in[\underline{\zeta}, \bar{\zeta}]$, the solution of $\dot{\hat{\zeta}}(t)=\operatorname{Proj}_{[\underline{\zeta}, \bar{\zeta}]}\{\tau\}$ remains in $[\underline{\zeta}, \bar{\zeta}]$;

(iii) If $\hat{\zeta} \in[\underline{\zeta}, \bar{\zeta}]$ and $\zeta \in[\underline{\zeta}, \bar{\zeta}]$, then $-\tilde{\zeta} \operatorname{Proj}_{[\underline{\zeta}, \bar{\zeta}]}\{\tau\} \leq-\tilde{\zeta} \tau$, where $\tilde{\zeta}=\zeta-\hat{\zeta}$.

Proposition 3.5. For the functions $\varphi_{1}(x, y, t), \varphi_{2}(x, t)$ and $\varphi_{3}(x, t)$ defined in (3.13), there exists a positive constant $M$ such that

$$
\left\{\begin{array}{l}
\int_{0}^{D} \int_{0}^{x} \varphi_{1}(x, y, t)^{2} \mathrm{~d} y \mathrm{~d} x \leq \frac{\sigma^{2} M^{2}}{W(t)^{2}}\left(\int_{0}^{D} w(x, t)^{2} \mathrm{~d} x\left(\int_{0}^{D} w_{x}(x, t)^{2} \mathrm{~d} x+\|X(t)\|^{2}\right)+\frac{1}{\rho^{2}}\|X(t)\|^{4}\right), \\
\int_{0}^{D}\left\|\varphi_{2}(x, t)\right\|^{2} \mathrm{~d} x \leq \frac{\sigma^{2} M^{2}}{W(t)^{2}}\left(\int_{0}^{D} w(x, t)^{2} \mathrm{~d} x\left(\int_{0}^{D} w_{x}(x, t)^{2} \mathrm{~d} x+\|X(t)\|^{2}\right)+\frac{1}{\rho^{2}}\|X(t)\|^{4}\right), \\
\int_{0}^{D} \varphi_{3}(x, t)^{2} \mathrm{~d} x \leq M^{2}\left(\int_{0}^{D} w_{x}(x, t)^{2} \mathrm{~d} x+\|X(t)\|^{2}\right) .
\end{array}\right.
$$

Proof. See part C of Appendix in the paper.

\section{Stability AnAlysis of The ClOSED-LOOP SYSTEM}

In this section, we show that the closed-loop system is stable in the desirable sense. For this, the following Lyapunov function is introduced:

$$
V(t)=\frac{1}{2} \log W(t)+\frac{\rho}{2 \sigma} \tilde{\varepsilon}(t)^{2}+\frac{\rho}{2 \sigma} \int_{0}^{D} \tilde{\lambda}(x, t)^{2} \mathrm{~d} x+\frac{\rho}{2 \sigma} \tilde{\theta}(t)^{\mathrm{T}} \tilde{\theta}(t),
$$

where $\sigma$ and $\rho$ are the same as in (3.20) and $W(t)$ has been defined by (3.21). 
Substituting (3.23) into the fourth equation of (3.12) yields $w(D, t)=0$. Then, computing the time derivative of $V(t)$ and using integration by parts, we have

$$
\begin{aligned}
& \dot{V}(t)=\frac{1}{W(t)}\left(\dot{X}(t)^{\mathrm{T}} P(\hat{\theta}) X(t)+X(t)^{\mathrm{T}} P(\hat{\theta}) \dot{X}(t)+\sum_{i=1}^{p} \dot{\hat{\theta}}_{i}(t) X(t)^{\mathrm{T}} \frac{\mathrm{d} P(\hat{\theta})}{\mathrm{d} \hat{\theta}_{i}} X(t)+\rho \int_{0}^{D} w(x, t) w_{t}(x, t) \mathrm{d} x\right) \\
& -\frac{\rho}{\sigma} \tilde{\varepsilon}(t) \dot{\hat{\varepsilon}}(t)-\frac{\rho}{\sigma} \int_{0}^{D} \tilde{\lambda}(x, t) \hat{\lambda}_{t}(x, t) \mathrm{d} x-\frac{\rho}{\sigma} \tilde{\theta}(t)^{\mathrm{T}} \dot{\hat{\theta}}(t) \\
& =\frac{1}{W(t)}\left(-X(t)^{\mathrm{T}} Q(\hat{\theta}) X(t)+2 X(t)^{\mathrm{T}} P(\hat{\theta}) B(\theta) w(0, t)+\sum_{i=1}^{p} \dot{\hat{\theta}}_{i}(t) X(t)^{\mathrm{T}} \frac{\mathrm{d} P(\hat{\theta})}{\mathrm{d} \hat{\theta}_{i}} X(t)\right. \\
& +2 X(t)^{\mathrm{T}} P(\hat{\theta})(\tilde{A}(\tilde{\theta})+\tilde{B}(\tilde{\theta}) K(\hat{\theta})) X(t)-\rho \varepsilon \int_{0}^{D} w_{x}(x, t)^{2} \mathrm{~d} x+\rho \int_{0}^{D} w(x, t) \varphi_{2}(x, t) \mathrm{d} x X(t) \\
& +\rho \int_{0}^{D} w(x, t) \int_{0}^{x} w(y, t) \varphi_{1}(x, y, t) \mathrm{d} y \mathrm{~d} x+\rho \int_{0}^{D} w(x, t)\left(\tilde{\lambda}(x, t)-\int_{0}^{x} \tilde{\lambda}(y, t) k(x, y, t) \mathrm{d} y\right) \mathrm{d} x C X(t) \\
& \left.+\rho \tilde{\varepsilon}(t) \int_{0}^{D} w(x, t) \varphi_{3}(x, t) \mathrm{d} x-\rho \int_{0}^{D} w(x, t) \gamma(x, t) \mathrm{d} x(\tilde{B}(\tilde{\theta}) w(0, t)+(\tilde{A}(\tilde{\theta})+\tilde{B}(\tilde{\theta}) K(\hat{\theta})) X(t))\right) \\
& -\frac{\rho}{\sigma} \tilde{\varepsilon}(t) \dot{\hat{\varepsilon}}(t)-\frac{\rho}{\sigma} \int_{0}^{D} \tilde{\lambda}(x, t) \hat{l}_{t}(x, t) \mathrm{d} x-\frac{\rho}{\sigma} \tilde{\theta}(t)^{\mathrm{T}} \dot{\hat{\theta}}(t) \\
& =\frac{1}{W(t)}\left(-X(t)^{\mathrm{T}} Q(\hat{\theta}) X(t)+2 X(t)^{\mathrm{T}} P(\hat{\theta}) B(\theta) w(0, t)+\sum_{i=1}^{p} \dot{\hat{\theta}}_{i}(t) X(t)^{\mathrm{T}} \frac{\mathrm{d} P(\hat{\theta})}{\mathrm{d} \hat{\theta}_{i}} X(t)\right. \\
& +2 \sum_{i=1}^{p} \tilde{\theta}_{i}(t) X(t)^{\mathrm{T}} P(\hat{\theta})\left(A_{i}+B_{i} K(\hat{\theta})\right) X(t)-\rho \varepsilon \int_{0}^{D} w_{x}(x, t)^{2} \mathrm{~d} x+\rho \int_{0}^{D} w(x, t) \varphi_{2}(x, t) \mathrm{d} x X(t) \\
& +\rho \int_{0}^{D} w(x, t) \int_{0}^{x} w(y, t) \varphi_{1}(x, y, t) \mathrm{d} y \mathrm{~d} x+\rho \int_{0}^{D} w(x, t)\left(\tilde{\lambda}(x, t)-\int_{0}^{x} \tilde{\lambda}(y, t) k(x, y, t) \mathrm{d} y\right) \mathrm{d} x C X(t) \\
& \left.+\rho \tilde{\varepsilon}(t) \int_{0}^{D} w(x, t) \varphi_{3}(x, t) \mathrm{d} x-\rho \int_{0}^{D} w(x, t) \gamma(x, t) \mathrm{d} x \sum_{i=1}^{p} \tilde{\theta}_{i}(t)\left(B_{i} w(0, t)+\left(A_{i}+B_{i} K(\hat{\theta})\right) X(t)\right)\right) \\
& -\frac{\rho}{\sigma} \tilde{\varepsilon}(t) \dot{\hat{\varepsilon}}(t)-\frac{\rho}{\sigma} \int_{0}^{D} \tilde{\lambda}(x, t) \hat{\lambda}_{t}(x, t) \mathrm{d} x-\frac{\rho}{\sigma} \sum_{i=1}^{p} \tilde{\theta}_{i}(t) \dot{\hat{\theta}}_{i}(t) .
\end{aligned}
$$

Changing the order of integration arrives at

$$
\int_{0}^{D} w(x, t) \int_{0}^{x} \tilde{\lambda}(y, t) k(x, y, t) \mathrm{d} y \mathrm{~d} x=\int_{0}^{D} \tilde{\lambda}(x, t) \int_{x}^{D} w(y, t) k(y, x, t) \mathrm{d} y \mathrm{~d} x .
$$

Substituting this and (3.20) into (4.1), and using claim (iii) of Proposition 3.4 and noting Assumption 2.1, we arrive at

$$
\begin{aligned}
\dot{V}(t)= & \frac{1}{W(t)}\left(-X(t)^{\mathrm{T}} Q(\hat{\theta}) X(t)+2 X(t)^{\mathrm{T}} P(\hat{\theta})\left(B_{0}+\sum_{i=1}^{p} \theta_{i} B_{i}\right) w(0, t)+\sum_{i=1}^{p} \dot{\hat{\theta}}_{i}(t) X(t)^{\mathrm{T}} \frac{\mathrm{d} P(\hat{\theta})}{\mathrm{d} \hat{\theta}_{i}} X(t)\right. \\
& \left.-\rho \varepsilon \int_{0}^{D} w_{x}(x, t)^{2} \mathrm{~d} x+\rho \int_{0}^{D} w(x, t) \int_{0}^{x} w(y, t) \varphi_{1}(x, y, t) \mathrm{d} y \mathrm{~d} x+\rho \int_{0}^{D} w(x, t) \varphi_{2}(x, t) \mathrm{d} x X(t)\right) \\
& +\rho \tilde{\varepsilon}(t) \frac{\tau_{\varepsilon}(t)-\dot{\hat{\varepsilon}}(t)}{\sigma}+\rho \int_{0}^{D} \tilde{\lambda}(x, t) \frac{\tau_{\lambda}(x, t)-\hat{\lambda}_{t}(x, t)}{\sigma} \mathrm{d} x+\rho \sum_{i=1}^{p} \tilde{\theta}_{i}(t) \frac{\tau_{\theta_{i}}(t)-\dot{\hat{\theta}}_{i}(t)}{\sigma} \\
\leq & \frac{1}{W(t)}\left(-X(t)^{\mathrm{T}} Q(\hat{\theta}) X(t)+2 X(t)^{\mathrm{T}} P(\hat{\theta})\left(B_{0}+\sum_{i=1}^{p} \theta_{i} B_{i}\right) w(0, t)+\sum_{i=1}^{p} \dot{\hat{\theta}}_{i}(t) X(t)^{\mathrm{T}} \frac{\mathrm{d} P(\hat{\theta})}{\mathrm{d} \hat{\theta}_{i}} X(t)\right. \\
& \left.-\rho \underline{\varepsilon} \int_{0}^{D} w_{x}(x, t)^{2} \mathrm{~d} x+\rho \int_{0}^{D} w(x, t) \int_{0}^{x} w(y, t) \varphi_{1}(x, y, t) \mathrm{d} y \mathrm{~d} x+\rho \int_{0}^{D} w(x, t) \varphi_{2}(x, t) \mathrm{d} x X(t)\right) .
\end{aligned}
$$


Then by (C.13) and Lemma D.4, it follows that

$$
\begin{aligned}
\dot{V}(t) \leq & \frac{1}{W(t)}\left(-\underline{\lambda}_{Q}\|X(t)\|^{2}+\frac{1}{2} \underline{\lambda}_{Q}\|X(t)\|^{2}+\frac{2 S_{P}^{2} M_{B}^{2}}{\underline{\lambda}_{Q}} w(0, t)^{2}+\sum_{i=1}^{p} \dot{\hat{\theta}}_{i}(t) X(t)^{\mathrm{T}} \frac{\mathrm{d} P(\hat{\theta})}{\mathrm{d} \hat{\theta}_{i}} X(t)\right. \\
& \left.-\rho \underline{\varepsilon} \int_{0}^{D} w_{x}(x, t)^{2} \mathrm{~d} x+\rho \int_{0}^{D} w(x, t) \int_{0}^{x} w(y, t) \varphi_{1}(x, y, t) \mathrm{d} y \mathrm{~d} x+\rho \int_{0}^{D} w(x, t) \varphi_{2}(x, t) \mathrm{d} x X(t)\right) \\
\leq & \frac{1}{W(t)}\left(-\frac{1}{2} \underline{\lambda}_{Q}\|X(t)\|^{2}-\left(\rho \underline{\varepsilon}-\frac{8 D S_{P}^{2} M_{B}^{2}}{\underline{\lambda}_{Q}}\right) \int_{0}^{D} w_{x}(x, t)^{2} \mathrm{~d} x+\sum_{i=1}^{p} \dot{\hat{\theta}}_{i}(t) X(t)^{\mathrm{T}} \frac{\mathrm{d} P(\hat{\theta})}{\mathrm{d} \hat{\theta}_{i}} X(t)\right. \\
& \left.+\rho \int_{0}^{D} w(x, t) \int_{0}^{x} w(y, t) \varphi_{1}(x, y, t) \mathrm{d} y \mathrm{~d} x+\rho \int_{0}^{D} w(x, t) \varphi_{2}(x, t) \mathrm{d} x X(t)\right),
\end{aligned}
$$

where $M_{B}=\left\|B_{0}\right\|+\sum_{i=1}^{p}\left\|B_{i}\right\| \cdot \max \left\{\left|\underline{\theta}_{i}\right|,\left|\bar{\theta}_{i}\right|\right\}, \underline{\lambda}_{Q}=\inf _{\hat{\theta} \in \Omega} \lambda_{\min }(Q(\hat{\theta}))\left(\operatorname{similarly}, \underline{\lambda}_{P}=\inf _{\hat{\theta} \in \Omega} \lambda_{\min }(P(\hat{\theta}))\right.$ is defined for later use).

Let $\rho>1$. By (C.12), (C.21) and noting that $\underline{\lambda}_{P}\|X(t)\|^{2} \leq X(t)^{\mathrm{T}} P(\hat{\theta}) X(t) \leq \frac{1}{2} W(t)$, we have

$$
\begin{aligned}
& \sum_{i=1}^{p} \dot{\hat{\theta}}_{i}(t) X(t)^{\mathrm{T}} \frac{\mathrm{d} P(\hat{\theta})}{\mathrm{d} \hat{\theta}_{i}} X(t) \leq \sum_{i=1}^{p} \sup _{\underline{\theta}_{i} \leq \theta_{i} \leq \bar{\theta}_{i}}\left\|\frac{\mathrm{d} P(\hat{\theta})}{\mathrm{d} \hat{\theta}_{i}}\right\|\|X(t)\|^{2}\left|\dot{\hat{\theta}}_{i}(t)\right| \\
& \leq \frac{\sigma}{W(t)} \sum_{i=1}^{p} \sup _{\underline{\theta}_{i} \leq \theta_{i} \leq \bar{\theta}_{i}}\left\|\frac{\mathrm{d} P(\hat{\theta})}{\mathrm{d} \hat{\theta}_{i}}\right\|\|X(t)\|^{2} \cdot\left(q_{1 i} \sqrt{\int_{0}^{D} w(x, t)^{2} \mathrm{~d} x} \sqrt{\int_{0}^{D} w_{x}(x, t)^{2} \mathrm{~d} x+\|X(t)\|^{2}}+\frac{q_{2 i}}{\rho}\|X(t)\|^{2}\right) \\
& \leq \frac{\sigma}{2 \underline{\lambda}_{P}} \sum_{i=1}^{p} \sup _{\underline{\theta}_{i} \leq \theta_{i} \leq \bar{\theta}_{i}}\left\|\frac{\mathrm{d} P(\hat{\theta})}{\mathrm{d} \hat{\theta}_{i}}\right\|\left(2 D q_{1 i}\left(\int_{0}^{D} w_{x}(x, t)^{2} \mathrm{~d} x+\|X(t)\|^{2}\right)+q_{2 i}\|X(t)\|^{2}\right) \\
& \leq \frac{\sigma}{2 \underline{\lambda}_{P}} \sum_{i=1}^{p}\left(2 D q_{1 i}+q_{2 i}\right) \sup _{\underline{\theta}_{i} \leq \theta_{i} \leq \bar{\theta}_{i}}\left\|\frac{\mathrm{d} P(\hat{\theta})}{\mathrm{d} \hat{\theta}_{i}}\right\|\left(\int_{0}^{D} w_{x}(x, t)^{2} \mathrm{~d} x+\|X(t)\|^{2}\right) \\
& \quad=\sigma d_{1}\left(\int_{0}^{D} w_{x}(x, t)^{2} \mathrm{~d} x+\|X(t)\|^{2}\right),
\end{aligned}
$$

where $d_{1}=\frac{1}{2 \underline{\lambda}_{P}} \sum_{i=1}^{p}\left(2 D q_{1 i}+q_{2 i}\right) \sup _{\underline{\theta}_{i} \leq \theta_{i} \leq \bar{\theta}_{i}}\left\|\frac{\mathrm{d} P(\hat{\theta})}{\mathrm{d} \hat{\theta}_{i}}\right\|$.

By Hölder's inequality, the first inequality of (3.24) and noting the general relation $\sqrt{a+b} \leq \sqrt{a}+\sqrt{b}$ for any positive real numbers $a$ and $b$, we have

$$
\begin{aligned}
& \rho \int_{0}^{D} w(x, t) \int_{0}^{x} w(y, t) \varphi_{1}(x, y, t) \mathrm{d} y \mathrm{~d} x \\
& \leq \rho \int_{0}^{D}|w(x, t)| \sqrt{\int_{0}^{x} \varphi_{1}(x, y, t)^{2} \mathrm{~d} y} \mathrm{~d} x \sqrt{\int_{0}^{D} w(y, t)^{2} \mathrm{~d} y} \\
& \leq \rho \int_{0}^{D} w(x, t)^{2} \mathrm{~d} x \sqrt{\int_{0}^{D} \int_{0}^{x} \varphi_{1}(x, y, t)^{2}} \mathrm{~d} y \mathrm{~d} x \\
& \leq \frac{\rho \sigma M}{W(t)} \int_{0}^{D} w(x, t)^{2} \mathrm{~d} x \sqrt{\int_{0}^{D} w(x, t)^{2}} \mathrm{~d} x \sqrt{\int_{0}^{D} w_{x}(x, t)^{2} \mathrm{~d} x+\|X(t)\|^{2}}+\frac{\sigma M}{W(t)} \int_{0}^{D} w(x, t)^{2} \mathrm{~d} x\|X(t)\|^{2} .
\end{aligned}
$$


Then, by (C.12) and nothing that $\rho \int_{0}^{D} w(x, t)^{2} \mathrm{~d} x \leq W(t)$ and $\underline{\lambda}_{P}\|X(t)\|^{2} \leq \frac{1}{2} W(t)$, it follows that

$$
\begin{aligned}
\rho \int_{0}^{D} w & (x, t) \int_{0}^{x} w(y, t) \varphi_{1}(x, y, t) \mathrm{d} y \mathrm{~d} x \\
& \leq 2 D \sigma M \sqrt{\int_{0}^{D} w_{x}(x, t)^{2} \mathrm{~d} x} \sqrt{\int_{0}^{D} w_{x}(x, t)^{2} \mathrm{~d} x+\|X(t)\|^{2}}+\frac{\sigma M}{W(t)} 4 D^{2} \int_{0}^{D} w_{x}(x, t)^{2} \mathrm{~d} x \frac{W(t)}{2 \underline{\lambda}_{P}} \\
& \leq 2 D \sigma M\left(1+\frac{D}{\underline{\lambda}_{P}}\right)\left(\int_{0}^{D} w_{x}(x, t)^{2} \mathrm{~d} x+\|X(t)\|^{2}\right) \\
& =\sigma d_{2}\left(\int_{0}^{D} w_{x}(x, t)^{2} \mathrm{~d} x+\|X(t)\|^{2}\right),
\end{aligned}
$$

where $d_{2}=2 D M\left(1+\frac{D}{\underline{\lambda}_{P}}\right)$. Similarly, by the second inequality of (3.24), we have

$$
\begin{aligned}
\rho \int_{0}^{D} w & (x, t) \varphi_{2}(x, t) X(t) \mathrm{d} x \\
& \leq \rho \sqrt{\int_{0}^{D} w(x, t)^{2} \mathrm{~d} x} \sqrt{\int_{0}^{D}\left|\varphi_{2}(x, t) X(t)\right|^{2} \mathrm{~d} x} \\
& \leq \rho \sqrt{\int_{0}^{D} w(x, t)^{2} \mathrm{~d} x} \sqrt{\int_{0}^{D}\left\|\varphi_{2}(x, t)\right\|^{2} \mathrm{~d} x\|X(t)\|} \\
& \leq \frac{\rho \sigma M}{W(t)} \int_{0}^{D} w(x, t)^{2} \mathrm{~d} x \sqrt{\int_{0}^{D} w_{x}(x, t)^{2} \mathrm{~d} x+\|X(t)\|^{2}}\|X(t)\|+\frac{\sigma M}{W(t)} \sqrt{\int_{0}^{D} w(x, t)^{2} \mathrm{~d} x}\|X(t)\|^{3} \\
& \leq \sigma M\left(\int_{0}^{D} w_{x}(x, t)^{2} \mathrm{~d} x+\|X(t)\|^{2}\right)+\frac{D \sigma M}{\underline{\lambda_{P}}} \sqrt{\int_{0}^{D} w_{x}(x, t)^{2} \mathrm{~d} x}\|X(t)\| \\
& \leq \sigma M\left(1+\frac{D}{\underline{\lambda}_{P}}\right)\left(\int_{0}^{D} w_{x}(x, t)^{2} \mathrm{~d} x+\|X(t)\|^{2}\right) \\
& =\sigma d_{3}\left(\int_{0}^{D} w_{x}(x, t)^{2} \mathrm{~d} x+\|X(t)\|^{2}\right),
\end{aligned}
$$

where $d_{3}=M\left(1+\frac{D}{\lambda_{P}}\right)$.

Substituting (4.3), (4.4) and (4.5) into (4.2) concludes that

$$
\dot{V}(t) \leq \frac{1}{W(t)}\left(-\left(\frac{1}{2} \underline{\lambda}_{Q}-\sigma\left(d_{1}+d_{2}+d_{3}\right)\right)\|X(t)\|^{2}-\left(\rho \underline{\varepsilon}-\frac{8 D S_{P}^{2} M_{B}^{2}}{\underline{\lambda}_{Q}}-\sigma\left(d_{1}+d_{2}+d_{3}\right)\right) \int_{0}^{D} w_{x}(x, t)^{2} \mathrm{~d} x\right) .
$$

Then, by choosing the design parameters $\rho$ and $\sigma$ as follows:

$$
\left\{\begin{array}{l}
\rho>\max \left\{\frac{8 D S_{P}^{2} M_{B}^{2}}{\frac{\varepsilon \lambda_{Q}}{Q}}, 1\right\}, \\
0<\sigma<\min \left\{\frac{1}{2} \underline{\lambda}_{Q}, \rho \underline{\varepsilon}-\frac{8 D S_{P}^{2} M_{B}^{2}}{\underline{\lambda}_{Q}}\right\} \cdot \frac{1}{d_{1}+d_{2}+d_{3}},
\end{array}\right.
$$

we arrive at

$$
\dot{V}(t) \leq-\frac{c}{W(t)}\left(\int_{0}^{D} w_{x}(x, t)^{2} \mathrm{~d} x+\|X(t)\|^{2}\right),
$$

where $c=\min \left\{\frac{1}{2} \underline{\lambda}_{Q}-\sigma\left(d_{1}+d_{2}+d_{3}\right), \rho \underline{\varepsilon}-\frac{8 D S_{P}^{2} M_{B}^{2}}{\underline{\lambda}_{Q}}-\sigma\left(d_{1}+d_{2}+d_{3}\right)\right\}$. 
We are now in a position to address the main results of the paper, which are summarized in the following theorem.

Theorem 4.1. Consider system (2.1) satisfying Assumptions 2.1 and 2.2 , and the initial value $u(x, 0)=u_{0}(x)$ such that $\int_{0}^{D} u_{0}(x)^{2} \mathrm{~d} x<+\infty$ and $\int_{0}^{D}\left(\frac{\mathrm{d} u_{0}(x)}{\mathrm{d} x}\right)^{2} \mathrm{~d} x<+\infty$, if $\rho$ and $\sigma$ are chosen to satisfy (4.6), then the adaptive controller consisting of (3.2), (3.19) and (3.23) guarantees that all the resulting closed-loop system states are bounded, and furthermore, $X(t)$ and $u(x, t)$ satisfy

$$
\lim _{t \rightarrow+\infty} X(t)=0, \quad \lim _{t \rightarrow+\infty} u(x, t)=0 .
$$

Proof. By Lemma 3.3, it suffices to show target system (3.12) under controller (3.23) satisfies $\lim _{t \rightarrow+\infty} U(t)=0$, $\sup _{t \geq 0} \int_{0}^{D} w_{x}(x, t)^{2} \mathrm{~d} x<+\infty$ and $\lim _{t \rightarrow+\infty}\left(\int_{0}^{D} w(x, t)^{2} \mathrm{~d} x+\|X(t)\|^{2}\right)=0$.

In order to prove the desirable stability of the closed-loop target system, the integrability of $\|X(t)\|^{2}$ and $\int_{0}^{D} w_{x}(x, t)^{2} \mathrm{~d} x$ are need to show. For this, by (3.2) and (3.11) and in the similar way to derive (3.16) and (3.17), we have

$$
\left\{\begin{array}{l}
\int_{0}^{D} w(x, t)^{2} \mathrm{~d} x \leq 3\left(1+D^{2} M_{1}^{2}\right) \int_{0}^{D} u(x, t)^{2} \mathrm{~d} x+3 D M_{1}^{2}\|X(t)\|^{2}, \\
\int_{0}^{D} w_{x}(x, t)^{2} \mathrm{~d} x \leq 3 \int_{0}^{D} u_{x}(x, t)^{2} \mathrm{~d} x+3 D^{2} M_{1}^{2} \int_{0}^{D} u(x, t)^{2} \mathrm{~d} x+3 D M_{1}^{2}\|X(t)\|^{2} .
\end{array}\right.
$$

Letting $t=0$ in above inequalities and noting that $\int_{0}^{D} u_{0}(x)^{2} \mathrm{~d} x<+\infty$ and $\int_{0}^{D}\left(\frac{\mathrm{d} u_{0}(x)}{\mathrm{d} x}\right)^{2} \mathrm{~d} x<+\infty$, we obtain that $\int_{0}^{D} w(x, 0)^{2} \mathrm{~d} x$ and $\int_{0}^{D} w_{x}(x, 0)^{2} \mathrm{~d} x$ are bounded, and hence $V(0)$ is bounded.

Integrating (4.7) over $[0, t]$ and $[0,+\infty)$, respectively, we have

$$
V(t) \leq V(0), \quad \int_{0}^{+\infty} \frac{\|X(t)\|^{2}+\int_{0}^{D} w_{x}(x, t)^{2} \mathrm{~d} x}{W(t)} \mathrm{d} t \leq \frac{V(0)}{c},
$$

which together with the definition of $V(t)$ imply that $\tilde{\theta}_{i}(t), \hat{\theta}_{i}(t), \tilde{\varepsilon}(t), \hat{\varepsilon}(t), \int_{0}^{D} \tilde{\lambda}(x, t)^{2} \mathrm{~d} x, \int_{0}^{D} \hat{\lambda}(x, t)^{2} \mathrm{~d} x,\|X(t)\|$ and $\int_{0}^{D} w(x, t)^{2} \mathrm{~d} x$ are bounded on $[0,+\infty)$, and hence $\|X(t)\|^{2}$ and $\int_{0}^{D} w_{x}(x, t)^{2} \mathrm{~d} x$ are integrable on $[0,+\infty)$.

By (3.12), integration by parts and noting that $w(D, t)=0$ under control (3.23), we have

$$
\begin{aligned}
\frac{1}{2} \frac{\mathrm{d}}{\mathrm{d} t} \int_{0}^{D} w_{x}(x, t)^{2} \mathrm{~d} x= & \int_{0}^{D} w_{x}(x, t) w_{x t}(x, t) \mathrm{d} x \\
= & -\varepsilon \int_{0}^{D} w_{x x}(x, t)^{2} \mathrm{~d} x-\int_{0}^{D} w_{x x}(x, t) \int_{0}^{x} w(y, t) \varphi_{1}(x, y, t) \mathrm{d} y \mathrm{~d} x \\
& -\int_{0}^{D} w_{x x}(x, t) \varphi_{2}(x, t) X(t) \mathrm{d} x-\tilde{\varepsilon}(t) \int_{0}^{D} w_{x x}(x, t) \varphi_{3}(x, t) \mathrm{d} x \\
& -\int_{0}^{D} w_{x x}(x, t)\left(\tilde{\lambda}(x, t)-\int_{0}^{x} \tilde{\lambda}(y, t) k(x, y, t) \mathrm{d} y\right) C X(t) \mathrm{d} x \\
& +\int_{0}^{D} w_{x x}(x, t) \gamma(x, t) \mathrm{d} x(\tilde{B}(\tilde{\theta}) w(0, t)+(\tilde{A}(\tilde{\theta})+\tilde{B}(\tilde{\theta}) K(\hat{\theta})) X(t)) .
\end{aligned}
$$

To show the boundedness of $\int_{0}^{D} w_{x}(x, t)^{2} \mathrm{~d} x$ on $[0,+\infty)$, the last five terms on the right-hand side of the above equations will be further handled. 
First, by Young's inequality, Hölder's inequality and the first inequality of (3.24), the second term on the right-hand side of the above equation satisfies:

$$
\begin{aligned}
&-\int_{0}^{D} w_{x x}(x, t) \int_{0}^{x} w(y, t) \varphi_{1}(x, y, t) \mathrm{d} y \mathrm{~d} x \\
& \leq \frac{\varepsilon}{5} \int_{0}^{D} w_{x x}(x, t)^{2} \mathrm{~d} x+\frac{5}{4 \varepsilon} \int_{0}^{D}\left(\int_{0}^{x} w(y, t) \varphi_{1}(x, y, t) \mathrm{d} y\right)^{2} \mathrm{~d} x \\
& \leq \frac{\varepsilon}{5} \int_{0}^{D} w_{x x}(x, t)^{2} \mathrm{~d} x+\frac{5}{4 \varepsilon} \int_{0}^{D} \int_{0}^{x} \varphi_{1}(x, y, t)^{2} \mathrm{~d} y \mathrm{~d} x \int_{0}^{D} w(x, t)^{2} \mathrm{~d} x \\
& \leq \frac{\varepsilon}{5} \int_{0}^{D} w_{x x}(x, t)^{2} \mathrm{~d} x+\frac{5 \sigma^{2} M^{2}}{4 \varepsilon W(t)^{2}}\left(\int_{0}^{D} w(x, t)^{2} \mathrm{~d} x\right)^{2}\left(\int_{0}^{D} w_{x}(x, t)^{2} \mathrm{~d} x+\|X(t)\|^{2}\right) \\
&+\frac{5 \sigma^{2} M^{2}}{4 \varepsilon \rho^{2} W(t)^{2}} \int_{0}^{D} w(x, t)^{2} \mathrm{~d} x\|X(t)\|^{4} \\
& \leq \frac{\varepsilon}{5} \int_{0}^{D} w_{x x}(x, t)^{2} \mathrm{~d} x+\frac{5 \sigma^{2} M^{2}}{4 \rho^{2} \varepsilon}\left(\int_{0}^{D} w_{x}(x, t)^{2} \mathrm{~d} x+\|X(t)\|^{2}\right)+\frac{5 \sigma^{2} M^{2}}{8 \varepsilon \rho^{3} \underline{\lambda}_{P}}\|X(t)\|^{2},
\end{aligned}
$$

where $\underline{\lambda}_{P}\|X(t)\|^{2} \leq \frac{1}{2} W(t)$ and $\rho \int_{0}^{D} w(x, t)^{2} \mathrm{~d} x \leq W(t)$ have been used. Similarly, by the second inequality of (3.24), the third term on the right-hand side of (4.8) satisfies:

$$
\begin{aligned}
&-\int_{0}^{D} w_{x x}(x, t) \varphi_{2}(x, t) X(t) \mathrm{d} x \\
& \leq \frac{\varepsilon}{5} \int_{0}^{D} w_{x x}(x, t)^{2} \mathrm{~d} x+\frac{5}{4 \varepsilon} \int_{0}^{D}\left|\varphi_{2}(x, t) X(t)\right|^{2} \mathrm{~d} x \\
& \leq \frac{\varepsilon}{5} \int_{0}^{D} w_{x x}(x, t)^{2} \mathrm{~d} x+\frac{5}{4 \varepsilon} \int_{0}^{D}\left\|\varphi_{2}(x, t)\right\|^{2} \mathrm{~d} x\|X(t)\|^{2} \\
& \leq \frac{\varepsilon}{5} \int_{0}^{D} w_{x x}(x, t)^{2} \mathrm{~d} x+\frac{5 \sigma^{2} M^{2}}{4 \varepsilon \rho^{2} W(t)^{2}}\|X(t)\|^{6} \\
&+\frac{5 \sigma^{2} M^{2}}{4 \varepsilon W(t)^{2}} \int_{0}^{D} w(x, t)^{2} \mathrm{~d} x\left(\int_{0}^{D} w_{x}(x, t)^{2} \mathrm{~d} x+\|X(t)\|^{2}\right)\|X(t)\|^{2} \\
& \leq \frac{\varepsilon}{5} \int_{0}^{D} w_{x x}(x, t)^{2} \mathrm{~d} x+\frac{5 \sigma^{2} M^{2}}{16 \varepsilon \rho^{2} \underline{\lambda}_{P}^{2}}\|X(t)\|^{2} \\
&+\frac{5 \sigma^{2} M^{2}}{8 \varepsilon \rho \underline{\lambda}_{P}}\left(\int_{0}^{D} w_{x}(x, t)^{2} \mathrm{~d} x+\|X(t)\|^{2}\right)
\end{aligned}
$$

and the fourth term on the right-hand side of (4.8) satisfies:

$$
\begin{aligned}
-\tilde{\varepsilon}(t) \int_{0}^{D} & w_{x x}(x, t) \varphi_{3}(x, t) \mathrm{d} x \\
& \leq \frac{\varepsilon}{5} \int_{0}^{D} w_{x x}(x, t)^{2} \mathrm{~d} x+\frac{5}{4 \varepsilon} \int_{0}^{D} \varphi_{3}(x, t)^{2} \mathrm{~d} x \sup _{t \geq 0} \tilde{\varepsilon}(t)^{2} \\
& \leq \frac{\varepsilon}{5} \int_{0}^{D} w_{x x}(x, t)^{2} \mathrm{~d} x+\frac{5 M^{2}}{4 \varepsilon}\left(\int_{0}^{D} w_{x}(x, t)^{2} \mathrm{~d} x+\|X(t)\|^{2}\right) \sup _{t \geq 0} \tilde{\varepsilon}(t)^{2} .
\end{aligned}
$$


Then, by (3.11) and Young's inequality, the fifth term on the right-hand side of (4.8) satisfies:

$$
\begin{aligned}
-\int_{0}^{D} w_{x x}(x, t)\left(\tilde{\lambda}(x, t)-\int_{0}^{x} \tilde{\lambda}(y, t) k(x, y, t) \mathrm{d} y\right) C X(t) \mathrm{d} x \\
\leq \frac{\varepsilon}{5} \int_{0}^{D} w_{x x}(x, t)^{2} \mathrm{~d} x+\frac{5}{4 \varepsilon} \int_{0}^{D}\left|\left(\tilde{\lambda}(x, t)-\int_{0}^{x} \tilde{\lambda}(y) k(x, y, t) \mathrm{d} y\right) C X(t)\right|^{2} \mathrm{~d} x \\
\leq \frac{\varepsilon}{5} \int_{0}^{D} w_{x x}(x, t)^{2} \mathrm{~d} x+\frac{5}{2 \varepsilon} \int_{0}^{D}\left(\tilde{\lambda}(x, t)^{2}+\int_{0}^{x} \tilde{\lambda}(y, t)^{2} \mathrm{~d} y \int_{0}^{x} k(x, y, t)^{2} \mathrm{~d} y\right) \mathrm{d} x\|C\|^{2}\|X(t)\|^{2} \\
\leq \frac{\varepsilon}{5} \int_{0}^{D} w_{x x}(x, t)^{2} \mathrm{~d} x+\frac{5}{2 \varepsilon} \int_{0}^{D} \tilde{\lambda}(x, t)^{2} \mathrm{~d} x\left(1+\int_{0}^{D} \int_{0}^{x} k(x, y, t)^{2} \mathrm{~d} y \mathrm{~d} x\right)\|C\|^{2}\|X(t)\|^{2} \\
\leq \frac{\varepsilon}{5} \int_{0}^{D} w_{x x}(x, t)^{2} \mathrm{~d} x+\mu_{1}\|X(t)\|^{2},
\end{aligned}
$$

where $\mu_{1}=\frac{5}{2 \varepsilon}\left(1+D^{2} M_{1}^{2}\right)\|C\|^{2} \cdot \sup _{t \geq 0} \int_{0}^{D} \tilde{\lambda}(x, t)^{2} \mathrm{~d} x$.

Finally, by Young's inequality and (C.13), the sixth term on the right-hand side of (4.8) should satisfy

$$
\begin{aligned}
\int_{0}^{D} w_{x x} & (x, t) \gamma(x, t) \mathrm{d} x(\tilde{B}(\tilde{\theta}) w(0, t)+(\tilde{A}(\tilde{\theta})+\tilde{B}(\tilde{\theta}) K(\hat{\theta})) X(t)) \\
\leq & \frac{\varepsilon}{5} \int_{0}^{D} w_{x x}(x, t)^{2} \mathrm{~d} x+\frac{5}{2 \varepsilon} \int_{0}^{D}|\gamma(x, t) \tilde{B}(\tilde{\theta})|^{2} \mathrm{~d} x w(0, t)^{2} \\
& +\frac{5}{2 \varepsilon} \int_{0}^{D}|\gamma(x, t)(\tilde{A}(\tilde{\theta})+\tilde{B}(\tilde{\theta}) K(\hat{\theta})) X(t)|^{2} \mathrm{~d} x \\
\leq & \frac{\varepsilon}{5} \int_{0}^{D} w_{x x}(x, t)^{2} \mathrm{~d} x+\frac{10}{\varepsilon} D^{2} M_{1}^{2}\left(\sum_{i=1}^{p}\left\|B_{i}\right\| \sup _{t \geq 0}\left|\tilde{\theta}_{i}(t)\right|\right)^{2} \int_{0}^{D} w_{x}(x, t)^{2} \mathrm{~d} x \\
& +\frac{5}{2 \varepsilon} D M_{1}^{2}\left(\sum_{i=1}^{p}\left(\left\|A_{i}\right\|+\left\|B_{i}\right\| S_{K}\right) \sup _{t \geq 0}\left|\tilde{\theta}_{i}(t)\right|\right)^{2}\|X(t)\|^{2} \\
\leq & \frac{\varepsilon}{5} \int_{0}^{D} w_{x x}(x, t)^{2} \mathrm{~d} x+\mu_{2}\left(\int_{0}^{D} w_{x}(x, t)^{2} \mathrm{~d} x+\|X(t)\|^{2}\right)
\end{aligned}
$$

where $\mu_{2}=\frac{10}{\varepsilon} D^{2} M_{1}^{2}\left(\sum_{i=1}^{p}\left\|B_{i}\right\| \sup _{t \geq 0}\left|\tilde{\theta}_{i}(t)\right|\right)^{2}+\frac{5}{2 \varepsilon} D M_{1}^{2}\left(\sum_{i=1}^{p}\left(\left\|A_{i}\right\|+\left\|B_{i}\right\| S_{K}\right) \sup _{t \geq 0}\left|\tilde{\theta}_{i}(t)\right|\right)^{2}$.

Substituting (4.9)-(4.13) into (4.8) yields

$$
\frac{1}{2} \frac{\mathrm{d}}{\mathrm{d} t} \int_{0}^{D} w_{x}(x, t)^{2} \mathrm{~d} x \leq \mu_{3}\left(\int_{0}^{D} w_{x}(x, t)^{2} \mathrm{~d} x+\|X(t)\|^{2}\right),
$$

where $\mu_{3}=\frac{5 \sigma^{2} M^{2}}{4 \rho \varepsilon}\left(1+\frac{1}{2 \rho \underline{\lambda}_{P}}\right)\left(\frac{1}{\rho}+\frac{1}{2 \underline{\lambda}_{P}}\right)+\frac{5 M^{2}}{4 \varepsilon} \sup _{t \geq 0} \tilde{\varepsilon}(t)^{2}+\mu_{1}+\mu_{2}$. Integrating the above inequality over $[0, t]$ concludes that

$$
\begin{aligned}
\int_{0}^{D} w_{x}(x, t)^{2} \mathrm{~d} x & \leq \int_{0}^{D} w_{x}(x, 0)^{2} \mathrm{~d} x+2 \mu_{3} \int_{0}^{t}\left(\int_{0}^{D} w_{x}(x, t)^{2} \mathrm{~d} x+\|X(t)\|^{2}\right) \mathrm{d} t \\
& \leq \int_{0}^{D} w_{x}(x, 0)^{2} \mathrm{~d} x+2 \mu_{3} \int_{0}^{+\infty}\left(\int_{0}^{D} w_{x}(x, t)^{2} \mathrm{~d} x+\|X(t)\|^{2}\right) \mathrm{d} t
\end{aligned}
$$

which shows that $\int_{0}^{D} w_{x}(x, t)^{2} \mathrm{~d} x$ is bounded on $[0,+\infty)$ since $\int_{0}^{D} w_{x}(x, 0)^{2} \mathrm{~d} x<+\infty$ and $\|X(t)\|^{2}$ and $\int_{0}^{D} w_{x}(x, t)^{2} \mathrm{~d} x$ are integrable on $[0,+\infty)$. 
We now turn to proving the convergence of $\int_{0}^{D} w(x, t)^{2} \mathrm{~d} x$. For this, by (3.12) and integration by parts, we have

$$
\begin{array}{rl}
\frac{1}{2} \frac{\mathrm{d}}{\mathrm{d} t} \int_{0}^{D} & w(x, t)^{2} \mathrm{~d} x=\int_{0}^{D} w(x, t) w_{t}(x, t) \mathrm{d} x \\
= & -\varepsilon \int_{0}^{D} w_{x}(x, t)^{2} \mathrm{~d} x+\int_{0}^{D} w(x, t) \int_{0}^{x} w(y, t) \varphi_{1}(x, y, t) \mathrm{d} y \mathrm{~d} x+\int_{0}^{D} w(x, t) \varphi_{2}(x, t) X(t) \mathrm{d} x \\
& +\tilde{\varepsilon}(t) \int_{0}^{D} w(x, t) \varphi_{3}(x, t) \mathrm{d} x+\int_{0}^{D} w(x, t)\left(\tilde{\lambda}(x, t)-\int_{0}^{x} \tilde{\lambda}(y, t) k(x, y, t) \mathrm{d} y\right) C X(t) \mathrm{d} x \\
& -\int_{0}^{D} w(x, t) \gamma(x, t) \mathrm{d} x(\tilde{B}(\tilde{\theta}) w(0, t)+(\tilde{A}(\tilde{\theta})+\tilde{B}(\tilde{\theta}) K(\hat{\theta})) X(t)) .
\end{array}
$$

In the similar way to derive (4.11), (4.12) and (4.13), the last three terms on the right-hand side of (4.14) satisfy

$$
\left\{\begin{array}{c}
\left|\tilde{\varepsilon}(t) \int_{0}^{D} w(x, t) \varphi_{3}(x, t) \mathrm{d} x\right| \leq \frac{\varepsilon}{5} \int_{0}^{D} w(x, t)^{2} \mathrm{~d} x+\frac{5 M^{2}}{4 \varepsilon}\left(\int_{0}^{D} w_{x}(x, t)^{2} \mathrm{~d} x+\|X(t)\|^{2}\right) \sup _{t \geq 0} \tilde{\varepsilon}(t)^{2}, \\
\left|\int_{0}^{D} w(x, t)\left(\tilde{\lambda}(x, t)-\int_{0}^{x} \tilde{\lambda}(y, t) k(x, y, t) \mathrm{d} y\right) C X(t) \mathrm{d} x\right| \leq \frac{\varepsilon}{5} \int_{0}^{D} w(x, t)^{2} \mathrm{~d} x+\mu_{1}\|X(t)\|^{2}, \\
\left|\int_{0}^{D} w(x, t) \gamma(x, t) \mathrm{d} x(\tilde{B}(\tilde{\theta}) w(0, t)+(\tilde{A}(\tilde{\theta})+\tilde{B}(\tilde{\theta}) K(\hat{\theta})) X(t))\right| \\
\quad \leq \frac{\varepsilon}{5} \int_{0}^{D} w(x, t)^{2} \mathrm{~d} x+\mu_{2}\left(\int_{0}^{D} w_{x}(x, t)^{2} \mathrm{~d} x+\|X(t)\|^{2}\right),
\end{array}\right.
$$

by which, (4.4) and (4.5), it follows from (4.14) that

$$
\begin{aligned}
\frac{1}{2}\left|\frac{\mathrm{d}}{\mathrm{d} t} \int_{0}^{D} w(x, t)^{2} \mathrm{~d} x\right| \leq & \frac{3 \varepsilon}{5} \int_{0}^{D} w(x, t)^{2} \mathrm{~d} x+\left(\varepsilon+\mu_{1}+\mu_{2}+\frac{5 M^{2}}{4 \varepsilon} \sup _{t \geq 0} \tilde{\varepsilon}(t)^{2}\right. \\
& \left.+\frac{\sigma\left(d_{2}+d_{3}\right)}{\rho}\right)\left(\|X(t)\|^{2}+\int_{0}^{D} w_{x}(x, t)^{2} \mathrm{~d} x\right)
\end{aligned}
$$

which implies that $\left|\frac{\mathrm{d}}{\mathrm{d} t} \int_{0}^{D} w(x, t)^{2} \mathrm{~d} x\right|$ is bounded on $[0,+\infty)$ since all the terms on the right-hand side of the above inequality are bounded on $[0,+\infty)$, and hence $\int_{0}^{D} w(x, t)^{2} \mathrm{~d} x$ is uniformly continuous on $[0,+\infty)$.

Noting that $w(D, t)=0$, by Poincaré's inequality, we have

$$
\int_{0}^{+\infty} \int_{0}^{D} w(x, t)^{2} \mathrm{~d} x \leq 4 D^{2} \int_{0}^{+\infty} \int_{0}^{D} w_{x}(x, t)^{2} \mathrm{~d} x<+\infty
$$

which shows that $\int_{0}^{D} w(x, t)^{2} \mathrm{~d} x$ is integrable on $[0,+\infty)$. This, together with the proven uniform continuity of $\int_{0}^{D} w(x, t)^{2} \mathrm{~d} x$ and the well known Barbălat's Lemma, directly yields $\lim _{t \rightarrow+\infty} \int_{0}^{D} w(x, t)^{2} \mathrm{~d} x=0$.

For the convergence of $X(t)$, by (3.12), (C.13) and Lemma D.4, there holds

$$
\begin{aligned}
& \left|\frac{\mathrm{d}}{\mathrm{d} t}\|X(t)\|^{2}\right| \\
& \quad=2\left|X(t)^{\mathrm{T}}(A(\hat{\theta})+B(\hat{\theta}) K(\hat{\theta})) X(t)\right|+2\left|X(t)^{\mathrm{T}}(\tilde{A}(\tilde{\theta})+\tilde{B}(\tilde{\theta}) K(\hat{\theta})) X(t)\right|+2\left|X(t)^{\mathrm{T}} B(\theta) w(0, t)\right| \\
& \quad \leq 2 S_{A+B K}\|X(t)\|^{2}+2 \sum_{i=1}^{p}\left(\left\|A_{i}\right\|+\left\|B_{i}\right\| S_{K}\right) \sup _{t \geq 0}\left|\tilde{\theta}_{i}(t)\right| \cdot\|X(t)\|^{2}+\|X(t)\|^{2}+4 D M_{B}^{2} \int_{0}^{D} w_{x}(x, t)^{2} \mathrm{~d} x,
\end{aligned}
$$

which shows that $\frac{\mathrm{d}}{\mathrm{d} t}\|X(t)\|^{2}$ is bounded on $[0,+\infty)$ due to the boundedness of $\|X(t)\|$ and $\int_{0}^{D} w_{x}(x, t)^{2} \mathrm{~d} x$ on $[0,+\infty)$, and hence $\|X(t)\|$ is uniformly continuous on $[0,+\infty)$. Then, noting that $\|X(t)\|^{2}$ has been proven to be integrable on $[0,+\infty)$, by Barbălat's Lemma, $\lim _{t \rightarrow+\infty} X(t)=0$ is directly obtained. 
Finally, we prove $\lim _{t \rightarrow+\infty} U(t)=0$. By (3.11), (3.23) Hölder's inequality and Lemma D.1, we have

$$
\begin{aligned}
|U(t)| \leq & \|X(t)\|\left(\|\gamma(D, t)\|+n \int_{0}^{D}\|k(D, y, t) \beta(y, t)\| \mathrm{d} y\right) \\
& +\sqrt{\int_{0}^{D} w(x, t)^{2} \mathrm{~d} x}\left(\sqrt{\int_{0}^{D} k(D, y, t)^{2} \mathrm{~d} y}+\sqrt{\int_{0}^{D}\left(\int_{y}^{D} k(D, \xi, t) l(\xi, y, t) \mathrm{d} \xi\right)^{2} \mathrm{~d} y}\right) \\
\leq & M_{1}\left(1+n D M_{1}\right)\|X(t)\|+\sqrt{D} M_{1}\left(1+D M_{1}\right) \sqrt{\int_{0}^{D} w(x, t)^{2} \mathrm{~d} x},
\end{aligned}
$$

which, together with the proven fact that $\lim _{t \rightarrow+\infty} X(t)=0$ and $\lim _{t \rightarrow+\infty} \int_{0}^{D} w(x, t)^{2} \mathrm{~d} x=0$, directly concludes $\lim _{t \rightarrow+\infty} U(t)=0$.

This completes the proof.

\section{Simulation ReSUlts}

In this section, numerical results are given to illustrate the effectiveness of the theoretical results for the following system:

$$
\left\{\begin{array}{l}
\dot{X}(t)=\theta X(t)+u(0, t), \\
u_{t}(x, t)=\varepsilon u_{x x}(x, t)+\lambda(x) X(t), \\
u_{x}(0, t)=0 \\
u(1, t)=U(t)
\end{array}\right.
$$

with scale initial condition $X_{0}=X(0)=20$ and $u_{0}(x)=u(x, 0)=50 \sin (2 \pi x)$. The unknown system parameters are supposed to be $\theta=3, \varepsilon=10, \lambda(x)=\frac{x}{2}$ with $\underline{\theta}=1, \bar{\theta}=4, \underline{\varepsilon}=1, \underline{\lambda}=0$ and $\bar{\lambda}=1$.

Letting $K(\hat{\theta})=-\hat{\theta}-2$ and $Q(\hat{\theta})=4 \hat{\theta}^{2}+4$, we obtain $P(\hat{\theta})=\hat{\theta}^{2}+1$ by solving Lyapunov equation (3.22). Moreover, by (4.6), we find suitable design parameters $\rho=300$ and $\sigma=1.5 \times 10^{-5}$. The initial values of the parameters updating laws are chosen as $\hat{\theta}(0)=2, \hat{\varepsilon}(0)=4$ and $\hat{\lambda}_{0}(x)=\frac{x}{4}$. It is necessary to point out that we do not calculate the series in (3.7) since the compact form of the sum of the infinite series is difficult to derive even for simple nonconstant parameter $\lambda(x)$ and more importantly, appropriate truncation of the series is sufficient for the practical implementation. Therefore, we replace $\gamma(x, t)$ by $\bar{\gamma}(x, t)=\sum_{i=0}^{50} \gamma_{i}(x, t)$ as its approximation in the controller. Hence, $\bar{k}(x, y, t)=\frac{1}{\hat{\varepsilon}(t)} \int_{0}^{x-y} \bar{\gamma}(\xi, t) \mathrm{d} \xi$. Then, by (3.2), (3.19) and (3.23), we have

$$
\left\{\begin{array}{l}
w(x, t)=u(x, t)-\int_{0}^{x} \bar{k}(x, y, t) u(y, t) \mathrm{d} y-\bar{\gamma}(x, t) X(t), \\
U(t)=\int_{0}^{1} \bar{k}(1, y, t) u(y, t) \mathrm{d} y+\bar{\gamma}(1, t) X(t), \\
\dot{\hat{\varepsilon}}(t)=\operatorname{Proj}_{[1,+\infty)}\left\{1.5 \times 10^{-5} \frac{\int_{0}^{1} w(x, t)\left(\bar{\gamma}(x, t)(w(0, t)-2 X(t))+\left(\int_{0}^{x} \bar{k}(x, y, t) \hat{\lambda}(y, t) \mathrm{d} y-\hat{\lambda}(x, t)\right) X(t)\right) \mathrm{d} x}{\hat{\varepsilon}(t)\left(1+2\left(1+\hat{\theta}(t)^{2}\right) X(t)^{2}+300 \int_{0}^{1} w(x, t)^{2} \mathrm{~d} x\right)}\right\}, \\
\hat{\lambda}_{t}(x, t)=\operatorname{Proj}_{[0,1]}\left\{1.5 \times 10^{-5} \frac{\left(w(x, t)-\int_{x}^{1} w(y, t) \bar{k}(y, x, t) \mathrm{d} y\right) X(t)}{1+2\left(1+\hat{\theta}(t)^{2}\right) X(t)^{2}+300 \int_{0}^{1} w(x, t)^{2} \mathrm{~d} x}\right\}, \\
\dot{\hat{\theta}(t)}=\operatorname{Proj}_{[1,4]}\left\{1.5 \times 10^{-5} \frac{\frac{1}{150}\left(1+\hat{\theta}(t)^{2}\right) X(t)^{2}-\int_{0}^{1} w(x, t) \bar{\gamma}(x, t) X(t) \mathrm{d} x}{1+2\left(1+\hat{\theta}(t)^{2}\right) X(t)^{2}+300 \int_{0}^{1} w(x, t)^{2} \mathrm{~d} x}\right\} .
\end{array}\right.
$$

Using the explicit forward Euler method (see, e.g., P. 406 of [19]) with 20-step discretization in space, five simulation figures are obtained for the closed-loop system signals. Specifically, Figures 1 and 2 show that closed-loop system states $u(x, t)$ and $X(t)$ ultimately converge to zero; Figure 3 shows that the parameter estimate $\hat{\varepsilon}(t)$ is always larger than $\underline{\varepsilon}$ and ultimately converges to a constant, Figure 4 shows that the parameter estimate $\hat{\theta}(t)$ always belong to interval $[\underline{\theta}, \bar{\theta}]$, and ultimately converges to a constant, Figure 5 shows that $\|\hat{\lambda}(x)\|=\left(\int_{0}^{1} \hat{\lambda}(x, t)^{2} \mathrm{~d} x\right)^{\frac{1}{2}}$ ultimately converges to a constant. 


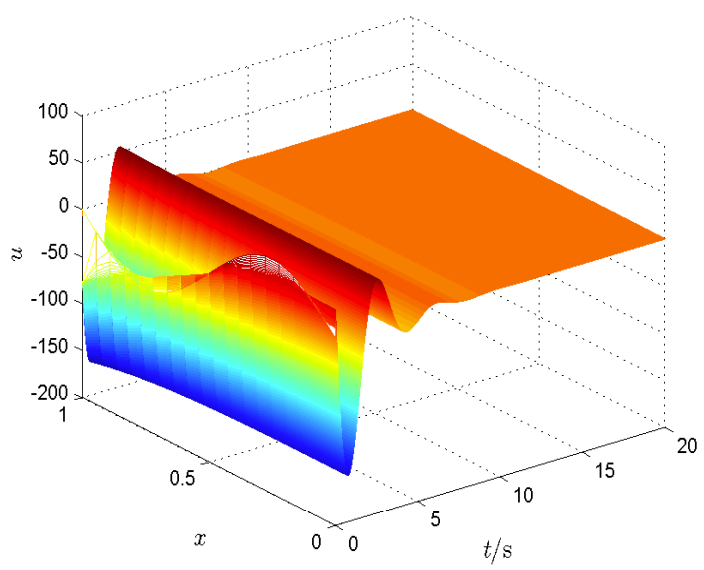

Figure 1. Trajectory of $u(x, t)$.

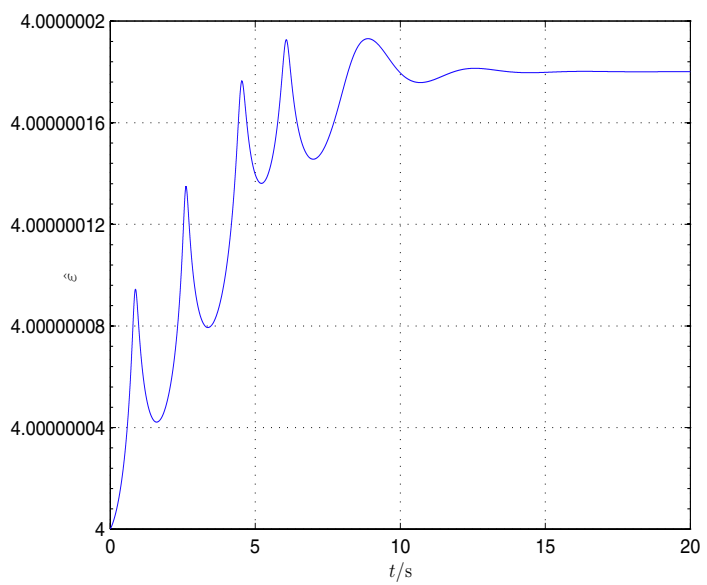

Figure 3 . Trajectory of $\hat{\varepsilon}(t)$.

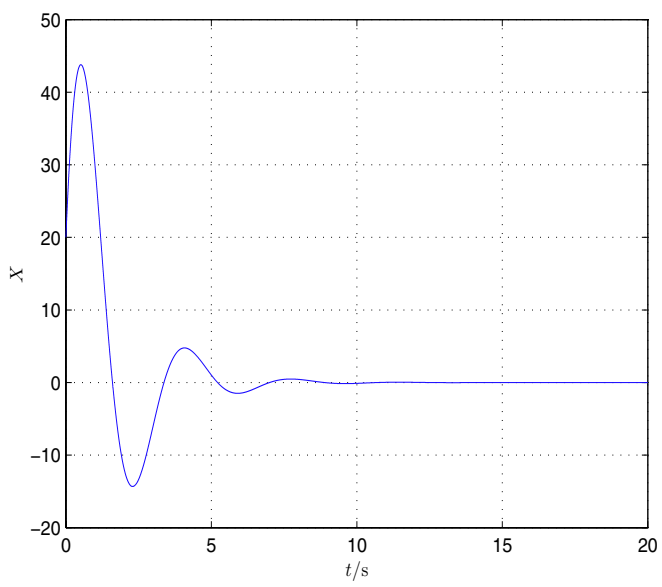

Figure 2. Trajectory of $X(t)$.

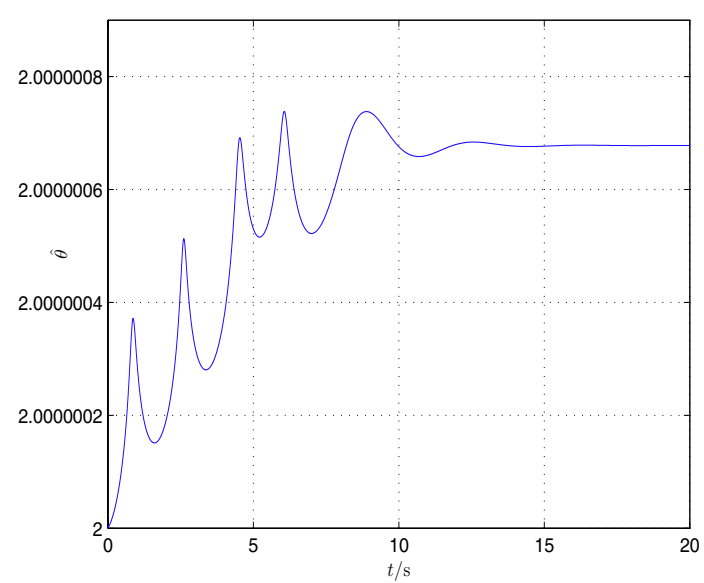

Figure 4. Trajectory of $\hat{\theta}(t)$.

\section{Concluding Remarks}

In this paper, adaptive stabilizing controller has been designed for a class of coupled PDE-ODE systems with multiple uncertainties. The essential difference between this paper and the existing related works is the the presence of the strong coupling and serious uncertainties. This makes the system under discussion more general and representative, and certainly more difficult to be stabilized. In the paper, a state-feedback adaptive stabilizing controller has been successfully constructed by utilizing infinite-dimensional backstepping method, adaptive techniques and certainty equivalence principle, and has been proven that the desirable control objective is surely established for the closed-loop system. It is worth pointing out that, the basic assumptions on the uncertainties are somewhat restrictive from a theoretical perspective, for instance $\theta_{i}$ 's in Assumption 2.1 are required to belong to a known interval. Therefore, how to relax these assumptions is quite meaningful and deserves further investigation. 


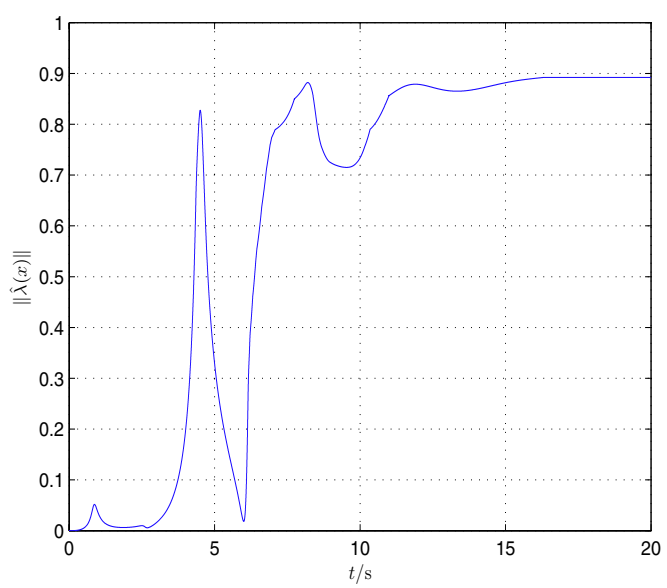

FiguRE 5. Trajectory of $\|\hat{\lambda}(x)\|$.

\section{A. Proof of Proposition 3.1}

To show the boundedness of the kernel functions and their partial derivatives with respect to $x$, we first estimate $\|\gamma(x, t)\|$ by induction, and then estimate others, such as $|k(x, y, t)|,\left\|\gamma_{x}(x, t)\right\|$ and $\left|k_{x}(x, y, t)\right|$.

For $i=0$, noting that $0 \leq x \leq D, \hat{\theta}(t) \in \Omega, \underline{\varepsilon} \leq \hat{\varepsilon}(t)$ and $\max _{0 \leq x \leq D, t \geq 0}|\hat{\lambda}(x, t)| \leq \lambda_{a}=\max \{|\underline{\lambda}|,|\bar{\lambda}|\}$, we have

$$
\begin{aligned}
\left\|\gamma_{0}(x, t)\right\| & \leq\|K(\hat{\theta})\|+\left\|\frac{1}{\hat{\varepsilon}(t)} \int_{0}^{x} \int_{0}^{\eta} \hat{\lambda}(\xi, t) C \mathrm{~d} \xi \mathrm{d} \eta\right\| \\
& \leq\|K(\hat{\theta})\|+\frac{1}{\underline{\varepsilon}} \int_{0}^{x} \int_{0}^{\eta}|\hat{\lambda}(\xi, t)| \cdot\|C\| \mathrm{d} \xi \mathrm{d} \eta \\
& \leq S_{K}+\frac{1}{\underline{\varepsilon}} D^{2} \lambda_{a}\|C\|=a_{0} .
\end{aligned}
$$

For $i \geq 1$, suppose that the following inequality holds

$$
\left\|\gamma_{i}(x, t)\right\| \leq a_{0} a_{1}^{i} \frac{x^{2 i}}{(2 i) !}
$$

with $a_{1}=\frac{n^{2}}{\underline{\varepsilon}}\left(S_{A}+\frac{D^{2} \lambda_{a}}{2 \underline{\varepsilon}} S_{B}\|C\|\right)$. Then, by Lemmas D.1 and (3.1), we have

$$
\begin{aligned}
\left\|\gamma_{i+1}(x, t)\right\| & \leq\left\|\frac{1}{\hat{\varepsilon}(t)} \int_{0}^{x} \int_{0}^{\eta} \gamma_{i}(\xi, t) A(\hat{\theta}) \mathrm{d} \xi \mathrm{d} \eta\right\|+\frac{1}{2}\left\|\frac{1}{\hat{\varepsilon}(t)^{2}} \int_{0}^{x} \int_{0}^{x-y}(x-y-\xi)^{2} \gamma_{i}(\xi, t) B(\hat{\theta}) \mathrm{d} \xi \hat{\lambda}(y, t) C \mathrm{~d} y\right\| \\
& \leq \frac{n^{2}}{\frac{\varepsilon}{n^{2}}} \int_{0}^{x} \int_{0}^{\eta}\left\|\gamma_{i}(\xi, t) A(\hat{\theta})\right\| \mathrm{d} \xi \mathrm{d} \eta+\frac{n^{2} D^{2}}{2 \underline{\varepsilon}^{2}} \int_{0}^{x} \int_{0}^{x-y}\left|\gamma_{i}(\xi, t) B(\hat{\theta})\right| \mathrm{d} \xi|\hat{\lambda}(y, t)| \cdot\|C\| \mathrm{d} y \\
& \leq \frac{n^{\varepsilon}}{\underline{\varepsilon}} S_{A} a_{0} a_{1}^{i} \int_{0}^{x} \int_{0}^{\eta} \frac{\xi^{2 i}}{2 i !} \mathrm{d} \xi \mathrm{d} \eta+\frac{n^{2} D^{2}}{2 \underline{\varepsilon}^{2}} S_{B}\|C\| \lambda_{a} a_{0} a_{1}^{i} \int_{0}^{x} \int_{0}^{x-y} \frac{\xi^{2 i}}{2 i !} \mathrm{d} \xi \mathrm{d} y \\
& =\frac{n^{2}}{\underline{\varepsilon}} S_{A} a_{0} a_{1}^{i} \frac{x^{2(i+1)}}{2(i+1) !}+\frac{n^{2} D^{2}}{2 \underline{\varepsilon}^{2}} S_{B}\|C\| \lambda_{a} a_{0} a_{1}^{i} \frac{x^{2(i+1)}}{2(i+1) !} \\
& =\frac{n^{2}}{\underline{\varepsilon}}\left(S_{A}+\frac{D^{2} \lambda_{a}}{2 \varepsilon} S_{B}\|C\|\right) a_{0} a_{1}^{i} \frac{x^{2(i+1)}}{2(i+1) !} \\
& =a_{0} a_{1}^{i+1} \frac{x^{2(i+1)}}{2(i+1) !},
\end{aligned}
$$


which implies the trueness of (A.1). Thus, by (3.3) and noting that $0 \leq x \leq D$, there holds

$$
\|\gamma(x, t)\| \leq \sum_{i=0}^{+\infty}\left\|\gamma_{i}(x, t)\right\| \leq \sum_{i=0}^{+\infty} a_{0} \frac{\left(D \sqrt{a_{1}}\right)^{2 i}}{(2 i) !} \leq a_{0} \exp \left(D \sqrt{a_{1}}\right) .
$$

By this and the second equation of (3.3), we arrive at

$$
|k(x, y, t)| \leq \frac{1}{\underline{\varepsilon}} \int_{0}^{x-y}|\gamma(\xi, t) B(\hat{\theta})| \mathrm{d} \xi \leq \frac{1}{\underline{\varepsilon}} a_{0} \exp \left(D \sqrt{a_{1}}\right) D S_{B},
$$

and

$$
\left|k_{x}(x, y, t)\right|=\left|\frac{1}{\hat{\varepsilon}(t)} \gamma(x-y, t) B(\hat{\theta})\right| \leq \frac{1}{\underline{\varepsilon}} a_{0} \exp \left(D \sqrt{a_{1}}\right) S_{B}
$$

By integrating both sides of the first equation of (3.4) over $[0, x]$ and noting $\gamma_{x}(0, t)=0$, we have

$$
\begin{aligned}
\gamma_{x}(x, t)= & -\frac{1}{\hat{\varepsilon}(t)} \int_{0}^{x} \hat{\lambda}(\xi, t) C \mathrm{~d} \xi+\frac{1}{\hat{\varepsilon}(t)} \int_{0}^{x} \gamma(\xi, t) A(\hat{\theta}) \mathrm{d} \xi \\
& +\frac{1}{\hat{\varepsilon}(t)^{2}} \int_{0}^{x} \int_{0}^{x-y}(x-y-\xi) \gamma(\xi, t) B(\hat{\theta}) \mathrm{d} \xi \hat{\lambda}(y, t) C \mathrm{~d} y .
\end{aligned}
$$

Then, there holds

$$
\left\|\gamma_{x}(x, t)\right\| \leq \frac{n D}{\underline{\varepsilon}}\left(\lambda_{a}\|C\|+a_{0} \exp \left(D \sqrt{a_{1}}\right)\left(S_{A}+\frac{n D^{2}}{\underline{\varepsilon}} \lambda_{a} S_{B}\|C\|\right)\right)=a_{2} .
$$

Moreover, by the first equation of (3.7) and noting that $0 \leq x \leq D, \underline{\varepsilon} \leq \hat{\varepsilon}(t)$ and $\max _{0 \leq x \leq D, t \geq 0}|\hat{\lambda}(x, t)| \leq \lambda_{a}$, we have

$$
\|\beta(x, t)\| \leq a_{3}, \quad\left\|\beta_{x}(x, t)\right\| \leq a_{3},
$$

with $a_{3}=\exp \left(D \max \left\{1, \frac{S_{A+B K}}{\underline{\varepsilon}}\right\}\right)\left(S_{K}+\frac{n D \lambda_{a}\|C\|}{\underline{\varepsilon}}\right)$, by which and the second equation of (3.7), it can be concluded that

$$
|l(x, y, t)| \leq \frac{1}{\underline{\varepsilon}} \int_{0}^{x-y}|\beta(\xi, t) B(\hat{\theta})| \mathrm{d} \xi \leq \frac{D a_{3} S_{B}}{\underline{\varepsilon}},
$$

and

$$
\left|l_{x}(x, y, t)\right|=\left|\frac{1}{\hat{\varepsilon}(t)} \beta(x-y, t) B(\hat{\theta})\right| \leq \frac{a_{3} S_{B}}{\underline{\varepsilon}} .
$$

Thus, choose $M_{1}=\max \left\{\max \left\{a_{3}, a_{0} \exp \left(D \sqrt{a_{1}}\right)\right\} \cdot \max \left\{1, \frac{D S_{B}}{\underline{\varepsilon}}, \frac{S_{B}}{\underline{\varepsilon}}\right\}, a_{2}\right\}$, which can upper bound the kernel functions on their separate domains of definition.

We next turn to show the trueness of (3.10). Substituting (3.6) into (3.2) yields

$$
\begin{aligned}
w(x, t)= & w(x, t)+\int_{0}^{x} l(x, y, t) w(y, t) \mathrm{d} y+\beta(x, t) X(t)-\gamma(x, t) X(t) \\
& -\int_{0}^{x} k(x, y, t)\left(w(y, t)+\int_{0}^{y} l(y, \xi, t) w(\xi, t) \mathrm{d} \xi+\beta(y, t) X(t)\right) \mathrm{d} y .
\end{aligned}
$$


By changing the order of integration, we have

$$
\int_{0}^{x} k(x, y, t) \int_{0}^{y} l(y, \xi, t) w(\xi, t) \mathrm{d} \xi \mathrm{d} y=\int_{0}^{x} \int_{y}^{x} k(x, \xi, t) l(\xi, y, t) \mathrm{d} \xi w(y, t) \mathrm{d} y .
$$

Substituting this into (A.2) and taking some managements yield

$$
\begin{aligned}
& \left(\gamma(x, t)-\beta(x, t)+\int_{0}^{x} k(x, y, t) \beta(y, t) \mathrm{d} y\right) X(t) \\
& =\int_{0}^{x}\left(l(x, y, t)-k(x, y, t)-\int_{y}^{x} k(x, \xi, t) l(\xi, y, t) \mathrm{d} \xi\right) w(y, t) \mathrm{d} y
\end{aligned}
$$

which implies (3.10) since the above equality holds for all $X(t)$ and $w(y, t)$.

\section{B. Proof of Proposition 3.2}

We will first show the first, third and fourth equations of (3.12), and then prove the third one.

Letting $x=0$ in $(3.2)$ and noting that $\gamma(0, t)=K(\hat{\theta})$, we have $u(0, t)=w(0, t)+K(\hat{\theta}) X(t)$. Substituting this into the first equation of (2.1) and noting that $A(\theta)=A(\hat{\theta})+\tilde{A}(\tilde{\theta})$ and $B(\theta)=B(\hat{\theta})+\tilde{B}(\tilde{\theta})$ yield

$$
\begin{aligned}
\dot{X}(t) & =A(\theta) X(t)+B(\theta) w(0, t)+B(\theta) K(\hat{\theta}) X(t) \\
& =(A(\hat{\theta})+B(\hat{\theta}) K(\hat{\theta})) X(t)+(\tilde{A}(\tilde{\theta})+\tilde{B}(\tilde{\theta}) K(\hat{\theta})) X(t)+B(\theta) w(0, t),
\end{aligned}
$$

which is the desired first equation of (3.12).

By computing $w_{x}(x, t)$ from (3.2) and letting $x=0$, we have

$$
w_{x}(0, t)=u_{x}(0, t)-k(0,0, t) u(0, t)-\gamma_{x}(0, t) X(t) .
$$

Then, by (3.4), (3.5) and noting that $u_{x}(0, t)=0$, we obtain $w_{x}(0, t)=0$, namely, the third equation of $(3.12)$.

Moreover, letting $x=D$ in $(3.2)$ and noting that $u(D, t)=U(t)$, we have

$$
w(D, t)=U(t)-\int_{0}^{D} k(D, y, t) u(y, t) \mathrm{d} y-\gamma(D, t) X(t) .
$$

Substituting (3.6) into the above equation and taking some simple managements conclude the fourth equation of (3.12).

We next turn to prove the second equation of (3.12). For this, we compute $w_{t}(x, t)$ from $(3.2$, that is,

$$
w_{t}(x, t)=u_{t}(x, t)-\int_{0}^{x} k(x, y, t) u_{t}(y, t) \mathrm{d} y-\gamma(x, t) \dot{X}(t)-\int_{0}^{x} k_{t}(x, y, t) u(y, t) \mathrm{d} y-\gamma_{t}(x, t) X(t) .
$$

All the terms on the right-hand side of (B.3) are not desirable and should be further handled.

Substituting the first equation of (3.12) into the third term on the right-hand side of (B.3), we have

$$
\begin{aligned}
-\gamma(x, t) \dot{X}(t)= & -\gamma(x, t) B(\theta) w(0, t)-\gamma(x, t)(A(\hat{\theta})+B(\hat{\theta}) K(\hat{\theta})) X(t) \\
& -\gamma(x, t)(\tilde{A}(\tilde{\theta})+\tilde{B}(\tilde{\theta}) K(\hat{\theta})) X(t),
\end{aligned}
$$

which is the desirable form.

In order to obtain the desirable expression of $u_{t}(x, t)$, we compute $u_{x}(x, t)$ and $u_{x x}(x, t)$ from (3.6). First, noting that $l(x, x, t)=0$, we have

$$
u_{x}(x, t)=w_{x}(x, t)+\int_{0}^{x} l_{x}(x, y, t) w(y, t) \mathrm{d} y+\beta_{x}(x, t) X(t) .
$$


By the second equation of (3.7) and noting $\beta(0, t)=K(\hat{\theta})$, we obtain $\left.l_{x}(x, y, t)\right|_{y=x}=\frac{1}{\hat{\varepsilon}(t)} K(\hat{\theta}) B(\hat{\theta})$. Then, by (3.8) and the above equation, we yield

$$
\begin{aligned}
u_{x x}(x, t)= & w_{x x}(x, t)+\left.l_{x}(x, y, t)\right|_{y=x} w(x, t)+\int_{0}^{x} l_{x x}(x, y, t) w(y, t) \mathrm{d} y+\beta_{x x}(x, t) X(t) \\
= & w_{x x}(x, t)+\frac{1}{\hat{\varepsilon}(t)} K(\hat{\theta}) B(\hat{\theta}) w(x, t)+\int_{0}^{x} l_{x x}(x, y, t) w(y, t) \mathrm{d} y \\
& +\frac{1}{\hat{\varepsilon}(t)} \beta(x, t)(A(\hat{\theta})+B(\hat{\theta}) K(\hat{\theta})) X(t)-\frac{1}{\hat{\varepsilon}(t)} \hat{\lambda}(x, t) C X(t) .
\end{aligned}
$$

Substituting this into the second equation of (2.1) and after some managements, we have

$$
\begin{aligned}
u_{t}(x, t)= & \varepsilon u_{x x}(x, t)+\lambda(x) C X(t) \\
= & \varepsilon w_{x x}(x, t)+\frac{\varepsilon}{\hat{\varepsilon}(t)} K(\hat{\theta}) B(\hat{\theta}) w(x, t)+\varepsilon \int_{0}^{x} l_{x x}(x, y, t) w(y, t) \mathrm{d} y \\
& +\frac{\varepsilon}{\hat{\varepsilon}(t)} \beta(x, t)(A(\hat{\theta})+B(\hat{\theta}) K(\hat{\theta})) X(t)+\left(\lambda(x)-\frac{\varepsilon}{\hat{\varepsilon}(t)} \hat{\lambda}(x, t)\right) C X(t) \\
= & \varepsilon w_{x x}(x, t)+\frac{\varepsilon}{\hat{\varepsilon}(t)} K(\hat{\theta}) B(\hat{\theta}) w(x, t)+\varepsilon \int_{0}^{x} l_{x x}(x, y, t) w(y, t) \mathrm{d} y \\
& +\frac{\varepsilon}{\hat{\varepsilon}(t)} \beta(x, t)(A(\hat{\theta})+B(\hat{\theta}) K(\hat{\theta})) X(t)+\tilde{\lambda}(x, t) C X(t)-\frac{\tilde{\varepsilon}(t)}{\hat{\varepsilon}(t)} \hat{\lambda}(x, t) C X(t),
\end{aligned}
$$

which is the desirable expression of the first term on the right-hand side of (B.3).

Substituting (B.5) into the second term on the right-hand side of (B.3), we have

$$
\begin{aligned}
-\int_{0}^{x} k(x, y, t) u_{t}(y, t) \mathrm{d} y= & -\varepsilon \int_{0}^{x} k(x, y, t) w_{y y}(y, t) \mathrm{d} y-\frac{\varepsilon}{\hat{\varepsilon}(t)} \int_{0}^{x} k(x, y, t) K(\hat{\theta}) B(\hat{\theta}) w(y, t) \mathrm{d} y \\
& -\varepsilon \int_{0}^{x} k(x, y, t) \int_{0}^{y} l_{y y}(y, \xi, t) w(\xi, t) \mathrm{d} \xi \mathrm{d} y \\
& -\frac{\varepsilon}{\hat{\varepsilon}(t)} \int_{0}^{x} k(x, y, t) \beta(y, t) \mathrm{d} y(A(\hat{\theta})+B(\hat{\theta}) K(\hat{\theta})) X(t) \\
& -\int_{0}^{x} k(x, y, t) \tilde{\lambda}(y, t) \mathrm{d} y C X(t)+\frac{\tilde{\varepsilon}(t)}{\hat{\varepsilon}(t)} \int_{0}^{x} k(x, y, t) \hat{\lambda}(y, t) \mathrm{d} y C X(t) .
\end{aligned}
$$

By the second equation of (3.3), we have $\left.k_{y}(x, y, t)\right|_{y=x}=-\frac{1}{\hat{\varepsilon}(t)} K(\hat{\theta}) B(\hat{\theta})$ and $\left.k_{y}(x, y, t)\right|_{y=0}=-\frac{1}{\hat{\varepsilon}(t)} \gamma(x, t) B(\hat{\theta})$, by which, (3.4), (3.5) and using integration by parts while noting the proven fact $w_{x}(0, t)=0$, the first term on the right-hand side of (B.6) satisfies

$$
\begin{array}{rl}
-\varepsilon \int_{0}^{x} & k(x, y, t) w_{y y}(y, t) \mathrm{d} y \\
& =-\varepsilon k(x, x, t) w_{x}(x, t)+\varepsilon k(x, 0, t) w_{y}(0, t)+\varepsilon \int_{0}^{x} k_{y}(x, y, t) w_{y}(y, t) \mathrm{d} y \\
& =\left.\varepsilon k_{y}(x, y, t)\right|_{y=x} w(x, t)-\left.\varepsilon k_{y}(x, y, t)\right|_{y=0} w(0, t)-\varepsilon \int_{0}^{x} k_{y y}(x, y, t) w(y, t) \mathrm{d} y \\
& =-\frac{\varepsilon}{\hat{\varepsilon}(t)} K(\hat{\theta}) B(\hat{\theta}) w(x, t)+\frac{\varepsilon}{\hat{\varepsilon}(t)} \gamma(x, t) B(\hat{\theta}) w(0, t)-\varepsilon \int_{0}^{x} k_{y y}(x, y, t) w(y, t) \mathrm{d} y .
\end{array}
$$

By computing the second-order partial derivatives of the first equation of (3.10) with respect to $y$, it can be concluded that

$$
\int_{y}^{x} k(x, \xi, t) l_{y y}(\xi, y, t) \mathrm{d} \xi=l_{y y}(x, y, t)-k_{y y}(x, y, t)-\frac{1}{\hat{\varepsilon}(t)} K(\hat{\theta}) B(\hat{\theta}) k(x, y, t),
$$


by which, (3.9) and changing the order of integration, the third term on the right-hand side of (B.6) satisfies

$$
\begin{array}{rl}
-\varepsilon \int_{0}^{x} & k(x, y, t) \int_{0}^{y} l_{y y}(y, \xi, t) w(\xi, t) \mathrm{d} \xi \mathrm{d} y \\
& =-\varepsilon \int_{0}^{x} w(y, t) \int_{y}^{x} k(x, \xi, t) l_{\xi \xi}(\xi, y, t) \mathrm{d} \xi \mathrm{d} y \\
& =-\varepsilon \int_{0}^{x} w(y, t) \int_{y}^{x} k(x, \xi, t) l_{y y}(\xi, y, t) \mathrm{d} \xi \mathrm{d} y \\
& =-\varepsilon \int_{0}^{x} w(y, t)\left(l_{y y}(x, y, t)-k_{y y}(x, y, t)-\frac{1}{\hat{\varepsilon}(t)} K(\hat{\theta}) B(\hat{\theta}) k(x, y, t)\right) \mathrm{d} y .
\end{array}
$$

Moreover, substituting the second equation of (3.10) into the fourth term of the right-hand side of (B.6), we yield

$$
-\frac{\varepsilon}{\hat{\varepsilon}(t)} \int_{0}^{x} k(x, y, t) \beta(y, t) \mathrm{d} y(A(\hat{\theta})+B(\hat{\theta}) K(\hat{\theta})) X(t)=-\frac{\varepsilon}{\hat{\varepsilon}(t)}(\beta(x, t)-\gamma(x, t))(A(\hat{\theta})+B(\hat{\theta}) K(\hat{\theta})) X(t)(\mathrm{B} .9)
$$

Hence, by substituting (B.7)-(B.9) into (B.6) and after some managements, we obtain the desirable form of the second term on the right-hand side of (B.3), that is,

$$
\begin{aligned}
-\int_{0}^{x} k(x, y, t) u_{t}(y, t) \mathrm{d} y & \\
= & -\frac{\varepsilon}{\hat{\varepsilon}(t)} K(\hat{\theta}) B(\hat{\theta}) w(x, t)+\frac{\varepsilon}{\hat{\varepsilon}(t)} \gamma(x, t) B(\hat{\theta}) w(0, t)-\varepsilon \int_{0}^{x} l_{y y}(x, y, t) w(y, t) \mathrm{d} y \\
& \quad-\frac{\varepsilon}{\hat{\varepsilon}(t)}(\beta(x, t)-\gamma(x, t))(A(\hat{\theta})+B(\hat{\theta}) K(\hat{\theta})) X(t)-\int_{0}^{x} k(x, y, t) \tilde{\lambda}(y, t) \mathrm{d} y C X(t) \\
& +\frac{\tilde{\varepsilon}(t)}{\hat{\varepsilon}(t)} \int_{0}^{x} k(x, y, t) \hat{\lambda}(y, t) \mathrm{d} y C X(t) .
\end{aligned}
$$

Computing the partial derivative of (3.10) with respect to $t$ yields

$$
\left\{\begin{aligned}
k_{t}(x, y, t)+\int_{y}^{x} k_{t}(x, \xi, t) l(\xi, y, t) \mathrm{d} \xi & =l_{t}(x, y, t)-\int_{y}^{x} k(x, \xi, t) l_{t}(\xi, y, t) \mathrm{d} \xi \\
\int_{0}^{x} k_{t}(x, y, t) \beta(y, t) \mathrm{d} y+\gamma_{t}(x, t) & =\beta_{t}(x, t)-\int_{0}^{x} k(x, y, t) \beta_{t}(y, t) \mathrm{d} y
\end{aligned}\right.
$$

by which and (3.6), we obtain the desirable form of the last two terms on the right-hand side of (B.3)

$$
\begin{aligned}
-\int_{0}^{x} & k_{t}(x, y, t) u(y, t) \mathrm{d} y-\gamma_{t}(x, t) X(t) \\
& =-\int_{0}^{x} k_{t}(x, y, t)\left(w(y, t)+\int_{0}^{y} l(y, \xi, t) w(\xi, t) \mathrm{d} \xi+\beta(y, t) X(t)\right) \mathrm{d} y-\gamma_{t}(x, t) X(t) \\
& =-\int_{0}^{x} w(y, t)\left(k_{t}(x, y, t)+\int_{y}^{x} k_{t}(x, \xi, t) l(\xi, y, t) \mathrm{d} \xi\right) \mathrm{d} y-\left(\int_{0}^{x} k_{t}(x, y, t) \beta(y, t) \mathrm{d} y+\gamma_{t}(x, t)\right) X(t) \\
& =-\int_{0}^{x} w(y, t)\left(l_{t}(x, y, t)-\int_{y}^{x} k(x, \xi, t) l_{t}(\xi, y, t) \mathrm{d} \xi\right) \mathrm{d} y-\left(\beta_{t}(x, t)-\int_{0}^{x} k(x, y, t) \beta_{t}(y, t) \mathrm{d} y\right) X(t) \\
& =\int_{0}^{x} w(y, t) \varphi_{1}(x, y, t) \mathrm{d} y+\varphi_{2}(x, t) X(t) .
\end{aligned}
$$


Substituting (B.4), (B.5), (B.10) and (B.11) into (B.3) and after some simple managements, we have

$$
\begin{aligned}
w_{t}(x, t)= & \varepsilon w_{x x}(x, t)+\int_{0}^{x} w(y, t) \varphi_{1}(x, y, t) \mathrm{d} y+\varphi_{2}(x, t) X(t)+\left(\tilde{\lambda}(x, t)-\int_{0}^{x} \tilde{\lambda}(y, t) k(x, y, t) \mathrm{d} y\right) C X(t) \\
& +\gamma(x, t)\left(\frac{\varepsilon}{\hat{\varepsilon}(t)} B(\hat{\theta})-B(\theta)\right) w(0, t)-\gamma(x, t)(\tilde{A}(\tilde{\theta})+\tilde{B}(\tilde{\theta}) K(\hat{\theta})) X(t) \\
& +\frac{\tilde{\varepsilon}(t)}{\hat{\varepsilon}(t)} \gamma(x, t)(A(\hat{\theta})+B(\hat{\theta}) K(\hat{\theta})) X(t)+\frac{\tilde{\varepsilon}(t)}{\hat{\varepsilon}(t)}\left(\int_{0}^{x} k(x, y) \hat{\lambda}(y, t) \mathrm{d} y-\hat{\lambda}(x, t)\right) C X(t) \\
= & \varepsilon w_{x x}(x, t)+\int_{0}^{x} w(y, t) \varphi_{1}(x, y, t) \mathrm{d} y+\varphi_{2}(x, t) X(t)+\left(\tilde{\lambda}(x, t)-\int_{0}^{x} \tilde{\lambda}(y, t) k(x, y, t) \mathrm{d} y\right) C X(t) \\
& -\gamma(x, t)(\tilde{B}(\tilde{\theta}) w(0, t)+(\tilde{A}(\tilde{\theta})+\tilde{B}(\tilde{\theta}) K(\hat{\theta})) X(t))+\tilde{\varepsilon}(t) \varphi_{3}(x, t),
\end{aligned}
$$

where $\varphi_{3}(\cdot)$ has been defined in (3.13). Thus we derive the second equation of (3.12).

\section{Proof of Proposition 3.5}

We will first prove the third inequality of (3.24) and then prove the other two ones.

By controller (3.23), we obtain $w(D, t)=0$. Then, by Poincaré's inequality and Agmon's inequality, we have

$$
\begin{aligned}
\int_{0}^{D} w(x, t)^{2} \mathrm{~d} x & \leq 4 D^{2} \int_{0}^{D} w_{x}(x, t)^{2} \mathrm{~d} x \\
w(0, t)^{2} & \leq 2 \sqrt{\int_{0}^{D} w(x, t)^{2} \mathrm{~d} x \int_{0}^{D} w_{x}(x, t)^{2} \mathrm{~d} x} \leq 4 D \int_{0}^{D} w_{x}(x, t)^{2} \mathrm{~d} x,
\end{aligned}
$$

by which and completing the square while noting that $\hat{\varepsilon}(t) \geq \underline{\varepsilon}$, there holds

$$
\begin{aligned}
\varphi_{3}(x, t)^{2} & \leq \frac{2}{\varepsilon^{2}}\left(\left\|\varphi_{4}(x, t)\right\|^{2}\|X(t)\|^{2}+|\gamma(x, t) B(\hat{\theta})|^{2} w(0, t)^{2}\right) \\
& \leq \frac{2}{\underline{\varepsilon}^{2}}\left(\left\|\varphi_{4}(x, t)\right\|^{2}\|X(t)\|^{2}+4 D|\gamma(x, t) B(\hat{\theta})|^{2} \int_{0}^{D} w_{x}(x, t)^{2} \mathrm{~d} x\right),
\end{aligned}
$$

where $\varphi_{4}(x, t)=\gamma(x, t)(A(\hat{\theta})+B(\hat{\theta}) K(\hat{\theta}))+\int_{0}^{x} k(x, y, t) \hat{\lambda}(y, t) \mathrm{d} y C-\hat{\lambda}(x, t) C$. Integrating both sides of the above inequality over $[0, D]$, we have

$$
\begin{aligned}
\int_{0}^{D} \varphi_{3}(x, t)^{2} \mathrm{~d} x & \leq \frac{2}{\underline{\varepsilon}^{2}}\left(\int_{0}^{D}\left\|\varphi_{4}(x, t)\right\|^{2} \mathrm{~d} x\|X(t)\|^{2}+4 D \int_{0}^{D}|\gamma(x, t) B(\hat{\theta})|^{2} \mathrm{~d} x \int_{0}^{D} w_{x}(x, t)^{2} \mathrm{~d} x\right) \\
& \leq m_{1}\left(\int_{0}^{D} w_{x}(x, t)^{2} \mathrm{~d} x+\|X(t)\|^{2}\right)
\end{aligned}
$$

where $m_{1}=\frac{2 D}{\varepsilon^{2}} \max \left\{\left(M_{1} S_{A+B K}+\lambda_{a}\|C\|\left(1+D M_{1}\right)\right)^{2}, 4 D M_{1}^{2} S_{B}^{2}\right\}$.

To prove the other two inequalities, the estimations of $\left\|\beta_{t}(x, t)\right\|$ and $\left|l_{t}(x, y, t)\right|$ are needed. We will realize this by estimating $|\dot{\hat{\varepsilon}}(t)|, \int_{0}^{D}\left|\hat{\lambda}_{t}(x, t)\right| \mathrm{d} x$ and $\left|\dot{\hat{\theta}}_{i}(t)\right|$. First, by claim (i) of Proposition 3.4, (3.20), (C.14) and Hölder's inequality, it follows from (3.19) that

$$
\begin{aligned}
|\dot{\hat{\varepsilon}}(t)| & \leq \frac{\sigma}{W(t)}\left|\int_{0}^{D} w(x, t) \varphi_{3}(x, t) \mathrm{d} x\right| \leq \frac{\sigma}{W(t)} \sqrt{\int_{0}^{D} w(x, t)^{2} \mathrm{~d} x \int_{0}^{D} \varphi_{3}(x, t)^{2} \mathrm{~d} x} \\
& \leq \frac{\sigma \sqrt{m_{1}}}{W(t)} \sqrt{\int_{0}^{D} w(x, t)^{2} \mathrm{~d} x} \sqrt{\int_{0}^{D} w_{x}(x, t)^{2} \mathrm{~d} x+\|X(t)\|^{2}} .
\end{aligned}
$$


Similarly, by (3.19) and noting that $0 \leq x \leq D$, we conclude

$$
\begin{aligned}
\int_{0}^{D}\left|\hat{\lambda}_{t}(x, t)\right| \mathrm{d} x & \leq \frac{\sigma}{W(t)}\left(\int_{0}^{D}|w(x, t)| \mathrm{d} x+\int_{0}^{D} \int_{x}^{D}|w(y, t) k(y, x, t)| \mathrm{d} y \mathrm{~d} x\right)|C X(t)| \\
& \leq \frac{\sigma \sqrt{D}}{W(t)} \sqrt{\int_{0}^{D} w(x, t)^{2} \mathrm{~d} x}\left(1+\sqrt{\int_{0}^{D} \int_{x}^{D} k(y, x, t)^{2} \mathrm{~d} y} \mathrm{~d} x\right)\|C\| \cdot\|X(t)\| \\
& \leq \frac{\sigma}{W(t)} m_{2} \sqrt{\int_{0}^{D} w(x, t)^{2} \mathrm{~d} x}\|X(t)\|,
\end{aligned}
$$

where $m_{2}=\sqrt{D}\|C\|\left(1+D M_{1}\right)$. Moreover, by (3.19) and claim (i) of Proposition 3.4, for $i=1, \ldots, p, \dot{\hat{\theta}}_{i}(t)$ satisfies

$$
\begin{aligned}
\left|\dot{\hat{\theta}}_{i}(t)\right| \leq & \frac{\sigma}{W(t)}\left|\int_{0}^{D} w(x, t) \gamma(x, t) \mathrm{d} x\left(B_{i} w(0, t)+\left(A_{i}+B_{i} K(\hat{\theta})\right)\right) X(t)\right| \\
& +\frac{2 \sigma}{\rho W(t)}\left|X(t)^{\mathrm{T}} P(\hat{\theta})\left(A_{i}+B_{i} K(\hat{\theta})\right) X(t)\right| \\
\leq & \frac{\sigma}{W(t)} \int_{0}^{D}\left|w(x, t) \gamma(x, t) B_{i} w(0, t)\right| \mathrm{d} x+\frac{\sigma}{W(t)} \int_{0}^{D}\left|w(x, t) \gamma(x, t)\left(A_{i}+B_{i} K(\hat{\theta})\right) X(t)\right| \mathrm{d} x \\
& +\frac{2 \sigma}{\rho W(t)}\left|X(t)^{\mathrm{T}} P(\hat{\theta})\left(A_{i}+B_{i} K(\hat{\theta})\right) X(t)\right| .
\end{aligned}
$$

By (3.11), (C.13) and Hölder's inequality, we yield

$$
\begin{aligned}
& \int_{0}^{D}\left|w(x, t) \gamma(x, t) B_{i} w(0, t)\right| \mathrm{d} x \leq \sqrt{\int_{0}^{D} w(x, t)^{2} \mathrm{~d} x} \sqrt{\int_{0}^{D}\left|\gamma(x, t) B_{i}\right|^{2} \mathrm{~d} x|w(0, t)|} \\
& \leq 2 D M_{1}\left\|B_{i}\right\| \sqrt{\int_{0}^{D} w(x, t)^{2} \mathrm{~d} x} \sqrt{\int_{0}^{D} w_{x}(x, t)^{2} \mathrm{~d} x} .
\end{aligned}
$$

Similarly, there holds

$$
\begin{aligned}
\int_{0}^{D}\left|w(x, t) \gamma(x, t)\left(A_{i}+B_{i} K(\hat{\theta})\right) X(t)\right| \mathrm{d} x & \leq \sqrt{\int_{0}^{D} w(x, t)^{2} \mathrm{~d} x} \sqrt{\int_{0}^{D}\left|\gamma(x, t)\left(A_{i}+B_{i} K(\hat{\theta})\right) X(t)\right|^{2} \mathrm{~d} x} \\
& \leq \sqrt{D} M_{1}\left(\left\|A_{i}\right\|+\left\|B_{i}\right\| S_{K}\right) \sqrt{\int_{0}^{D} w(x, t)^{2} \mathrm{~d} x\|X(t)\| .}
\end{aligned}
$$

Moreover, it can be verified that

$$
\left|X(t)^{\mathrm{T}} P(\hat{\theta})\left(A_{i}+B_{i} K(\hat{\theta})\right) X(t)\right| \leq S_{P}\left(\left\|A_{i}\right\|+\left\|B_{i}\right\| S_{K}\right)\|X(t)\|^{2} .
$$

Substituting (C.18)-(C.20) into (C.17) directly yields

$$
\left|\dot{\hat{\theta}}_{i}(t)\right| \leq \frac{\sigma q_{1 i}}{W(t)} \sqrt{\int_{0}^{D} w(x, t)^{2} \mathrm{~d} x} \sqrt{\int_{0}^{D} w_{x}(x, t)^{2} \mathrm{~d} x+\|X(t)\|^{2}}+\frac{\sigma q_{2 i}}{\rho W(t)}\|X(t)\|^{2},
$$

where $q_{1 i}=\sqrt{D} M_{1}\left(2 \sqrt{D}\left\|B_{i}\right\|+\left\|A_{i}\right\|+\left\|B_{i}\right\| S_{K}\right)$ and $q_{2 i}=2 S_{P}\left(\left\|A_{i}\right\|+\left\|B_{i}\right\| S_{K}\right), i=1, \ldots, p$. 
Letting $G(x, t)=\left[\begin{array}{c}0 \frac{A(\hat{\theta})+B(\hat{\theta}) K(\hat{\theta})}{\hat{\varepsilon}(t)} \\ I\end{array}\right]$ and $E(x, t)=\exp (G(\hat{\varepsilon}, \hat{\theta}) x)$. Then, it can be verified that

$$
\|G(x, t)\| \leq \max \left\{1, \frac{S_{A+B K}}{\underline{\varepsilon}}\right\},\|E(x, t)\| \leq \exp \left(D \max \left\{1, \frac{S_{A+B K}}{\underline{\varepsilon}}\right\}\right)=E_{A+B K} .
$$

Moreover, by (2.2), there holds

$$
H(\hat{\theta})=\frac{\mathrm{d}}{\mathrm{d} t}(A(\hat{\theta})+B(\hat{\theta}) K(\hat{\theta}))=\sum_{i=1}^{p}\left(A_{i}+B_{i} K(\hat{\theta})+B(\hat{\theta}) \frac{\mathrm{d} K(\hat{\theta})}{\mathrm{d} \hat{\theta}_{i}}\right) \dot{\hat{\theta}}_{i}(t) .
$$

Hence, we have

$$
G_{t}(\hat{\varepsilon}, \hat{\theta})=\left[\begin{array}{l}
0-\frac{A(\hat{\theta})+B(\hat{\theta}) K(\hat{\theta})}{\hat{\varepsilon}(t)^{2}} \dot{\hat{\varepsilon}}(t)+\frac{1}{\hat{\varepsilon}(t)} H(\hat{\theta}) \\
0
\end{array}\right]
$$

by which,

$$
\left\|G_{t}(\hat{\varepsilon}, \hat{\theta})\right\| \leq \frac{S_{A+B K}}{\underline{\varepsilon}^{2}}|\dot{\hat{\varepsilon}}(t)|+\sum_{i=1}^{p} r_{i}\left|\dot{\hat{\theta}}_{i}(t)\right|,
$$

where $r_{i}=\frac{1}{\underline{\varepsilon}}\left(\left\|A_{i}\right\|+\left\|B_{i}\right\| S_{K}+S_{B} \sup _{\underline{\theta}_{i} \leq \theta_{i} \leq \bar{\theta}_{i}}\left\|\frac{\mathrm{d} K(\hat{\theta})}{\mathrm{d} \hat{\theta}_{i}}\right\|\right), i=1, \ldots, p$. This, together with the above expression of $E(x, t)$, yields

$$
\begin{aligned}
\left\|E_{t}(x, t)\right\| & \leq D \exp (D\|G(\hat{\varepsilon}, \hat{\theta})\|)\left\|G_{t}(\hat{\varepsilon}, \hat{\theta})\right\| \\
& \leq D \exp \left(D \max \left\{1, \frac{S_{A+B K}}{\underline{\varepsilon}}\right\}\right)\left(\frac{S_{A+B K}}{\underline{\varepsilon}^{2}}|\dot{\hat{\varepsilon}}(t)|+\sum_{i=1}^{p} r_{i}\left|\dot{\hat{\theta}}_{i}(t)\right|\right) \\
& \leq m_{3}\left(|\dot{\hat{\varepsilon}}(t)|+\sum_{i=1}^{p} r_{i}\left|\dot{\hat{\theta}}_{i}(t)\right|\right),
\end{aligned}
$$

where $m_{3}=D E_{A+B K} \max \left\{\frac{S_{A+B K}}{\underline{\varepsilon}^{2}}, 1\right\}$.

With the above expression of $E(x, t)$ in mind, we re-express $\beta(x, t)$ as

$$
\beta(x, t)=K(\hat{\theta})\left[\begin{array}{ll}
I & 0
\end{array}\right] E(x, t)\left[\begin{array}{ll}
I & 0
\end{array}\right]^{\mathrm{T}}+\int_{0}^{x}\left[0-\frac{\hat{\lambda}(\xi, t) C}{\hat{\varepsilon}(t)}\right] E(x-\xi, t)\left[\begin{array}{ll}
I & 0
\end{array}\right]^{\mathrm{T}} \mathrm{d} \xi .
$$

Computing the partial derivative with respect to $t$ yields

$$
\begin{aligned}
& \beta_{t}(x, t)=\sum_{i=1}^{p} \frac{\mathrm{d} K(\hat{\theta})}{\mathrm{d} \hat{\theta}_{i}} \dot{\hat{\theta}}_{i}(t)\left[\begin{array}{ll}
I & 0
\end{array}\right] E(x, t)\left[\begin{array}{ll}
I & 0
\end{array}\right]^{\mathrm{T}}+K(\hat{\theta})\left[\begin{array}{ll}
I & 0
\end{array}\right] E_{t}(x, t)\left[\begin{array}{ll}
I & 0
\end{array}\right]^{\mathrm{T}} \\
& +\int_{0}^{x}\left[0 \frac{\dot{\hat{\varepsilon}}(t) \hat{\lambda}(\xi, t) C}{\hat{\varepsilon}(t)^{2}}\right] E(x-\xi, t)\left[\begin{array}{ll}
I & 0
\end{array}\right]^{\mathrm{T}} \mathrm{d} \xi+\int_{0}^{x}\left[0-\frac{\hat{\lambda}_{t}(\xi, t) C}{\hat{\varepsilon}(t)}\right] E(x-\xi, t)\left[\begin{array}{ll}
I & 0
\end{array}\right]^{\mathrm{T}} \mathrm{d} \xi \\
& +\int_{0}^{x}\left[0-\frac{\hat{\lambda}(\xi, t) C}{\hat{\varepsilon}(t)}\right] E_{t}(x-\xi, t)\left[\begin{array}{ll}
I & 0
\end{array}\right]^{\mathrm{T}} \mathrm{d} \xi
\end{aligned}
$$


by which, Lemma D.1 and (C.15), (C.16), (C.21), (C.22) and (C.23), we have

$$
\begin{aligned}
& \left\|\beta_{t}(x, t)\right\| \leq \sum_{i=1}^{p}\left\|\frac{\mathrm{d} K(\hat{\theta})}{\mathrm{d} \hat{\theta}_{i}}\right\| \cdot\left|\dot{\hat{\theta}}_{i}(t)\right| \cdot\|E(x, t)\|+\|K(\hat{\theta})\| \cdot\left\|E_{t}(x, t)\right\|+\frac{1}{\underline{\varepsilon}^{2}} n \lambda_{a}\|C\| \cdot|\dot{\hat{\varepsilon}}(t)| \int_{0}^{x}\|E(x-\xi, t)\| \mathrm{d} \xi \\
& +\frac{1}{\underline{\varepsilon}} n\|C\| \cdot \sup _{0 \leq \xi \leq x, t \geq 0}\|E(x-\xi, t)\| \int_{0}^{D}\left|\hat{\lambda}_{t}(\xi, t)\right| \mathrm{d} \xi+\frac{1}{\underline{\varepsilon}} n \lambda_{a}\|C\| \int_{0}^{x}\left\|E_{t}(x-\xi, t)\right\| \mathrm{d} \xi \\
& \leq m_{4}\left(|\dot{\hat{\varepsilon}}(t)|+\int_{0}^{D}\left|\hat{\lambda}_{t}(x, t)\right| \mathrm{d} x+\sum_{i=1}^{p} s_{i}\left|\dot{\hat{\theta}}_{i}(t)\right|\right) \\
& \leq \frac{\sigma m_{4}\left(\sqrt{m_{1}}+m_{2}+\sum_{i=1}^{p} q_{1 i} s_{i}\right)}{W(t)} \sqrt{\int_{0}^{D} w(x, t)^{2} \mathrm{~d} x} \sqrt{\int_{0}^{D} w_{x}(x, t)^{2} \mathrm{~d} x+\|X(t)\|^{2}} \\
& +\frac{\sigma m_{4}}{\rho W(t)} \sum_{i=1}^{p} q_{2 i} s_{i}\|X(t)\|^{2} \\
& \leq \frac{\sigma \delta_{1}}{W(t)} \sqrt{\int_{0}^{D} w(x, t)^{2} \mathrm{~d} x} \sqrt{\int_{0}^{D} w_{x}(x, t)^{2} \mathrm{~d} x+\|X(t)\|^{2}}+\frac{\sigma \delta_{2}}{\rho W(t)}\|X(t)\|^{2},
\end{aligned}
$$

where $s_{i}=\sup _{\underline{\theta}_{i} \leq \theta_{i} \leq \bar{\theta}_{i}}\left\|\frac{\mathrm{d} K(\hat{\theta})}{\mathrm{d} \hat{\theta}_{i}}\right\| E_{A+B K}+S_{K} m_{3} r_{i}+\frac{n}{\underline{\varepsilon}} \lambda_{a}\|C\| D m_{3} r_{i}, i=1, \ldots, p, m_{4}=\max \left\{1, \frac{n}{\underline{\varepsilon}}\|C\| E_{A+B K}\right.$, $\left.S_{K} m_{3}+\frac{n}{\underline{\varepsilon}} \lambda_{a}\|C\| D\left(\frac{E_{A+B K}}{\underline{\varepsilon}}+m_{3}\right)\right\}, \delta_{1}=m_{4}\left(\sqrt{m_{1}}+m_{2}+\sum_{i=1}^{p} q_{1 i} s_{i}\right)$ and $\delta_{2}=m_{4} \sum_{i=1}^{p} q_{2 i} s_{i}$.

Moreover, by computing the time derivative of the second equality of (3.7), we have

$$
l_{t}(x, y, t)=-\frac{\dot{\hat{\varepsilon}}(t)}{\hat{\varepsilon}(t)^{2}} \int_{0}^{x-y} \beta(\xi, t) B(\hat{\theta}) \mathrm{d} \xi+\frac{1}{\hat{\varepsilon}(t)} \int_{0}^{x-y} \beta_{t}(\xi, t) B(\hat{\theta}) \mathrm{d} \xi+\frac{1}{\hat{\varepsilon}(t)} \int_{0}^{x-y} \beta(\xi, t) \mathrm{d} \xi \sum_{i=1}^{p} B_{i} \dot{\hat{\theta}}_{i}(t) .
$$

Then, by (3.11), (C.15), (C.21) and the second inequality of (C.24), there holds

$$
\begin{aligned}
\left|l_{t}(x, y, t)\right| \leq & m_{7}\left(|\dot{\hat{\varepsilon}}(t)|+\int_{0}^{D}\left|\hat{\lambda}_{t}(x, t)\right| \mathrm{d} x+\sum_{i=1}^{p} v_{i}\left|\dot{\hat{\theta}}_{i}(t)\right|\right) \\
\leq & \frac{\sigma m_{7}\left(\sqrt{m_{1}}+m_{2}+\sum_{i=1}^{p} q_{1 i} v_{i}\right)}{W(t)} \sqrt{\int_{0}^{D} w(x, t)^{2} \mathrm{~d} x} \sqrt{\int_{0}^{D} w_{x}(x, t)^{2} \mathrm{~d} x+\|X(t)\|^{2}} \\
& +\frac{\sigma m_{7}}{\rho W(t)} \sum_{i=1}^{p} q_{2 i} v_{i}\|X(t)\|^{2} \\
\leq & \frac{\sigma \delta_{3}}{W(t)} \sqrt{\int_{0}^{D} w(x, t)^{2} \mathrm{~d} x} \sqrt{\int_{0}^{D} w_{x}(x, t)^{2} \mathrm{~d} x+\|X(t)\|^{2}}+\frac{\sigma \delta_{4}}{\rho W(t)}\|X(t)\|^{2},
\end{aligned}
$$

with $v_{i}=\frac{D}{\underline{\varepsilon}}\left(S_{B} m_{4} s_{i}+M_{1}\left\|B_{i}\right\|\right), i=1, \ldots, p, m_{7}=\max \left\{\frac{D}{\underline{\varepsilon}} S_{B}\left(\frac{M_{1}}{\underline{\varepsilon}}+m_{4}\right), \frac{D}{\underline{\varepsilon}} S_{B} m_{4}, 1\right\}, \delta_{3}=m_{7}\left(\sqrt{m_{1}}+m_{2}+\right.$ $\left.\sum_{i=1}^{p} q_{1 i} v_{i}\right)$ and $\delta_{4}=m_{7} \sum_{i=1}^{p} q_{2 i} v_{i}$.

We are in a position to show the first two inequalities of (3.24). By (3.11) and completing the square, it follows from the first equality of (3.13) that

$$
\begin{aligned}
\varphi_{1}(x, y, t)^{2} & \leq 2 l_{t}(x, y, t)^{2}+2\left(\int_{y}^{x} k(x, \xi, t) l_{t}(\xi, y, t) \mathrm{d} \xi\right)^{2} \\
& \leq 2 l_{t}(x, y, t)^{2}+2 D^{2} \sup _{0 \leq y \leq \xi \leq x} l_{t}(\xi, y, t)^{2} \cdot \sup _{0 \leq y \leq \xi \leq x} k(x, \xi, t)^{2} \\
& \leq 2\left(1+D^{2} \sup _{0 \leq \xi \leq x, t \geq 0} k(x, \xi, t)^{2}\right) \sup _{0 \leq y \leq x \leq D} l_{t}(x, y, t)^{2}
\end{aligned}
$$




$$
\leq 2\left(1+D^{2} M_{1}^{2}\right) \sup _{0 \leq y \leq x \leq D} l_{t}(x, y, t)^{2} .
$$

By (C.25) and integrating the above inequality over $[0, x]$ with respect to $y$ and then $[0, D]$ with respect to $x$, we yield

$$
\begin{aligned}
\int_{0}^{D} \int_{0}^{x} \varphi_{1}(x, y, t)^{2} \mathrm{~d} y \mathrm{~d} x & \leq 2 D^{2}\left(1+D^{2} M_{1}^{2}\right) \sup _{0 \leq y \leq x \leq D} l_{t}(x, y, t)^{2} \\
& \leq \frac{\sigma^{2} m_{8}}{W(t)^{2}} \int_{0}^{D} w(x, t)^{2} \mathrm{~d} x\left(\int_{0}^{D} w_{x}(x, t)^{2} \mathrm{~d} x+\|X(t)\|^{2}\right)+\frac{\sigma^{2} m_{9}}{\rho^{2} W(t)^{2}}\|X(t)\|^{4},
\end{aligned}
$$

where $m_{8}=4 D^{2}\left(1+D^{2} M_{1}^{2}\right) \delta_{3}^{2}, m_{9}=4 D^{2}\left(1+D^{2} M_{1}^{2}\right) \delta_{4}^{2}$.

Moreover, by (3.11), the second equality of (3.13) and Lemma D.1, we obtain

$$
\begin{aligned}
\left\|\varphi_{2}(x, t)\right\|^{2} & \leq 2\left\|\beta_{t}(x, t)\right\|^{2}+2\left\|\int_{0}^{x} k(x, y, t) \beta_{t}(y, t) \mathrm{d} y\right\|^{2} \\
& \leq 2\left\|\beta_{t}(x, t)\right\|^{2}+2 n^{2} D^{2} \sup _{0 \leq y \leq x \leq D} k(x, y, t)^{2} \cdot \sup _{0 \leq y \leq x \leq D}\left\|\beta_{t}(y, t)\right\|^{2} \\
& \leq 2\left(1+n^{2} D^{2} \sup _{0 \leq y \leq x \leq D} k(x, y, t)^{2}\right) \sup _{0 \leq x \leq D}\left\|\beta_{t}(x, t)\right\|^{2} \\
& \leq 2\left(1+n^{2} D^{2} M_{1}^{2}\right) \sup _{0 \leq x \leq D}\left\|\beta_{t}(x, t)\right\|^{2} .
\end{aligned}
$$

Integrating the above inequality over $[0, D]$ and using (C.24) yield

$$
\begin{aligned}
\int_{0}^{D}\left\|\varphi_{2}(x, t)\right\|^{2} \mathrm{~d} x & \leq 2 D\left(1+n^{2} D^{2} M_{1}^{2}\right) \sup _{0 \leq x \leq D}\left\|\beta_{t}(x, t)\right\|^{2} \\
& \leq \frac{\sigma^{2} m_{10}}{W(t)^{2}} \int_{0}^{D} w(x, t)^{2} \mathrm{~d} x\left(\int_{0}^{D} w_{x}(x, t)^{2} \mathrm{~d} x+\|X(t)\|^{2}\right)+\frac{\sigma^{2} m_{11}}{\rho^{2} W(t)^{2}}\|X(t)\|^{4},
\end{aligned}
$$

where $m_{10}=4 D\left(1+n^{2} D^{2} M_{1}^{2}\right) \delta_{1}^{2}, m_{11}=4 D\left(1+n^{2} D^{2} M_{1}^{2}\right) \delta_{2}^{2}$.

Finally, choosing $M=\sqrt{\max \left\{m_{1}, m_{8}, m_{9}, m_{10}, m_{11}\right\}}$ directly concludes (3.24).

\section{Useful INEQUalities}

Lemma D.1. For any matrix function $A(x)=\left(a_{i j}(x)\right):[0, D] \rightarrow \mathbb{R}^{m \times n}$ which is continuous and integrable on $[0, D]$, the following inequality holds:

$$
\left\|\int_{0}^{D} A(x) \mathrm{d} x\right\| \leq \sqrt{m} n \int_{0}^{D}\|A(x)\| \mathrm{d} x .
$$

Proof. Let $\|A(x)\|_{F}=\sqrt{\operatorname{tr}\left(A(x)^{\mathrm{T}} A(x)\right)}$. Then noting that $\lambda_{i}\left(A(x)^{\mathrm{T}} A(x)\right) \geq 0, i=1, \ldots, n$, we have

$$
\|A(x)\|=\sqrt{\lambda_{\max }\left(A(x)^{\mathrm{T}} A(x)\right)} \leq \sqrt{\sum_{i=1}^{n} \lambda_{i}\left(A(x)^{\mathrm{T}} A(x)\right)}=\sqrt{\operatorname{tr}\left(A(x)^{\mathrm{T}} A(x)\right)}=\|A(x)\|_{F} .
$$

Moreover,

$$
\|A(x)\|_{F}=\sqrt{\sum_{i=1}^{n} \lambda_{i}\left(A(x)^{\mathrm{T}} A(x)\right)} \leq \sqrt{n \lambda_{\max }\left(A(x)^{\mathrm{T}} A(x)\right)}=\sqrt{n}\|A(x)\| .
$$

Therefore by (D.26), we have

$$
\left\|\int_{0}^{D} A(x) \mathrm{d} x\right\|^{2} \leq\left\|\int_{0}^{D} A(x) \mathrm{d} x\right\|_{F}^{2}=\sum_{i=1}^{m} \sum_{j=1}^{n}\left|\int_{0}^{D} a_{i j}(x) \mathrm{d} x\right|^{2},
$$


by which, and noting that $\left|\int_{0}^{D} a_{i j}(x) \mathrm{d} x\right| \leq \int_{0}^{D}\left|a_{i j}(x)\right| \mathrm{d} x$, after some direct calculations, we obtain

$$
\begin{aligned}
\left\|\int_{0}^{D} A(x) \mathrm{d} x\right\|^{2} & \leq \sum_{i=1}^{m} \sum_{j=1}^{n}\left(\int_{0}^{D}\left|a_{i j}(x)\right| \mathrm{d} x\right)^{2} \\
& \leq\left(\sum_{i=1}^{m} \sum_{j=1}^{n} \int_{0}^{D}\left|a_{i j}(x)\right| \mathrm{d} x\right)^{2}=\left(\int_{0}^{D} \sum_{i=1}^{m} \sum_{j=1}^{n}\left|a_{i j}(x)\right| \mathrm{d} x\right)^{2} \\
& \leq m n\left(\int_{0}^{D} \sqrt{\sum_{i=1}^{m} \sum_{j=1}^{n}\left|a_{i j}(x)\right|^{2}} \mathrm{~d} x\right)^{2}=m n\left(\int_{0}^{D}\|A(x)\|_{F} \mathrm{~d} x\right)^{2} .
\end{aligned}
$$

Substituting (D.27) into the above inequality yields

$$
\left\|\int_{0}^{D} A(x) \mathrm{d} x\right\|^{2} \leq m n^{2}\left(\int_{0}^{D}\|A(x)\| \mathrm{d} x\right)^{2},
$$

which directly implies the desirable inequality.

Lemma D.2. (see [20]) (Poincaré's inequality) For any $w \in \mathbb{C}^{1}[0, D]$, there hold

$$
\left\{\begin{array}{l}
\int_{0}^{D} w(x)^{2} \mathrm{~d} x \leq 2 D w(0)^{2}+4 D^{2} \int_{0}^{D} w_{x}(x)^{2} \mathrm{~d} x \\
\int_{0}^{D} w(x)^{2} \mathrm{~d} x \leq 2 D w(D)^{2}+4 D^{2} \int_{0}^{D} w_{x}(x)^{2} \mathrm{~d} x .
\end{array}\right.
$$

Lemma D.3. (see [20]) (Agmon's inequality) For any $w \in \mathbb{C}^{1}[0, D]$, there hold

$$
\left\{\begin{array}{l}
w(x)^{2} \leq w(0)^{2}+2 \sqrt{\int_{0}^{D} w(x)^{2} \mathrm{~d} x \int_{0}^{D} w_{x}(x)^{2} \mathrm{~d} x} \\
w(x)^{2} \leq w(D)^{2}+2 \sqrt{\int_{0}^{D} w(x)^{2} \mathrm{~d} x \int_{0}^{D} w_{x}(x)^{2} \mathrm{~d} x} .
\end{array}\right.
$$

Lemma D.4. (see [21]) For any $x, y \in \mathbb{R}^{n}$ and any positive definite matrix $P \in \mathbb{R}^{n \times n}$, there holds

$$
2 x^{\mathrm{T}} y \leq x^{\mathrm{T}} P x+y^{\mathrm{T}} P^{-1} y .
$$

\section{REFERENCES}

[1] M. Krstić and A. Smyshlyaev, Backstepping boundary control for first order hyperbolic PDEs and application to systems with actuator and sensor delays. Syst. Control Lett. 57 (2008) 750-758.

[2] D.B. Pietri and M. Krstić, Adaptive trajectory tracking despite unknown input delay and plant parameters. Automatica 45 (2009) 2074-2081.

[3] M. Krstić, Compensating a string PDE in the actuation or sensing path of an unstable ODE. IEEE Trans. Automatic Control 54 (2009) 1362-1368.

[4] G.A. Susto and M. Krstić, Control of PDE-ODE cascades with Neumann interconnections. J. Franklin Institute 347 (2010) 284-314.

[5] M. Krstić, Compensating actuator and sensor dynamics governed by diffusion PDEs. Syst. Control Lett. 58 (2009) $372-377$.

[6] J. Li and Y.G. Liu, Adaptive control of the ODE systems with uncertain diffusion-dominated actuator dynamics. Internat. J. Control 85 (2012) 868-879.

[7] S.X. Tang and C.K. Xie, Stabilization for a coupled PDE-ODE control system. J. Franklin Institute 348 (2011) $2142-2155$.

[8] S.X. Tang and C.K. Xie, State and output feedback boundary control for a coupled PDE-ODE system. Syst. Control Lett. 60 (2011) 540-545.

[9] Z.C. Zhou and S.X. Tang, Boundary stabilization of a coupled wave-ODE system. Proc. Chinese Control Conf. Yantai, China (2011) 1048-1052.

[10] A.A. Masoud and S.A. Masoud, A self-organizing, hybrid PDE-ODE structure for motion control in informationally-deprived situations. Proc. IEEE Conf. Decision Control. Tampa, Florida, USA (1998) 2535-2540.

[11] C.F. Baicu, C.D. Rahn and D.M. Dawson, Backstepping boundary control of flexible-link electrically driven gantry robots. IEEE/ASME Trans. Mechatr. 3 (1998) 60-66.

[12] O. Morgul, Orientation and stabilization of a flexible beam attached to a rigid body: planar motion. IEEE Trans. Autom. Control 36 (1991) 953-962.

[13] D.M. Dawson, J.J. Carroll and M. Schneider, Integrator backstepping control of a brush DC motor turning a robotic load. IEEE Trans. Control Syst. Technol. 2 (1994) 233-244. 
[14] D.B. Chentouf, A note on stabilization of a hybrid PDE-ODE system. Proc. IEEE Conf. Decision Control. Orlando, Florida, USA (2001) 137-142.

[15] B. d'Andrea-Novel and J.M. Coron, Exponential stabilization of an overhead crane with flexible cable via a back-stepping approach. Automatica 36 (2000) 587-593.

[16] B. d'Andrea-Novel, F. Boustany, F. Conrad and B.P. Rao, Feedback stabilization of a hybrid PDE-ODE systems: application to an overhead crane. Math. Control, Signals, Syst. 7 (1994) 1-22.

[17] C. Panjapornpon, P. Limpanachaipornkul and T. Charinpanitkul, Control of coupled PDEs-ODEs using input-output linearization: application to cracking furnace. Chemical Engrg. Sci. 75 (2012) 144-151.

[18] M. Krstić and A. Smyshlyaev, Adaptive boundary control for unstable parabolic PDEs-part I: Lyapunov design. IEEE Trans. Automatic Control 53 (2008) 1575-1591.

[19] W.Y. Yang, W. Cao, T.S. Chung and J. Morris, Appl. Numer. Methods Using Matlab. John Wiley \& Sons, Hoboken, New Jersey (2005).

[20] K.D. Do and J. Pan, Boundary control of three-dimensional inextensible marine risers. J. Sound and Vibration 327 (2009) 299-321.

[21] Y.Y. Cao and J. Lam, Robust $\mathrm{H}^{\infty}$ control of uncertain markovian jump systems with time-delay. IEEE Trans. Autom. Control 45 (2000) $77-83$. 